Article

\title{
Quantitative Analysis of Polarimetric Model-Based Decomposition Methods
}

\author{
Qinghua Xie ${ }^{1,2}$, J. David Ballester-Berman ${ }^{2}$, Juan M. Lopez-Sanchez ${ }^{2, *}$, Jianjun Zhu ${ }^{1}$ \\ and Changcheng Wang ${ }^{1}$ \\ 1 School of Geosciences and Info-Physics, Central South University, Changsha 410083, China; \\ csuxqh@csu.edu.cn (Q.X.); zjj@csu.edu.cn (J.Z.); wangchangcheng@csu.edu.cn (C.W.) \\ 2 Institute for Computing Research (IUII), University of Alicante, Alicante E-03080, Spain; davidb@ua.es \\ * Correspondence: juanma.lopez@ua.es; Tel.: +34-96-590-9597
}

Academic Editors: Xiaofeng Li and Prasad S. Thenkabail

Received: 7 September 2016; Accepted: 16 November 2016; Published: 25 November 2016

\begin{abstract}
In this paper, we analyze the robustness of the parameter inversion provided by general polarimetric model-based decomposition methods from the perspective of a quantitative application. The general model and algorithm we have studied is the method proposed recently by Chen et al., which makes use of the complete polarimetric information and outperforms traditional decomposition methods in terms of feature extraction from land covers. Nevertheless, a quantitative analysis on the retrieved parameters from that approach suggests that further investigations are required in order to fully confirm the links between a physically-based model (i.e., approaches derived from the Freeman-Durden concept) and its outputs as intermediate products before any biophysical parameter retrieval is addressed. To this aim, we propose some modifications on the optimization algorithm employed for model inversion, including redefined boundary conditions, transformation of variables, and a different strategy for values initialization. A number of Monte Carlo simulation tests for typical scenarios are carried out and show that the parameter estimation accuracy of the proposed method is significantly increased with respect to the original implementation. Fully polarimetric airborne datasets at L-band acquired by German Aerospace Center's (DLR's) experimental synthetic aperture radar (E-SAR) system were also used for testing purposes. The results show different qualitative descriptions of the same cover from six different model-based methods. According to the Bragg coefficient ratio (i.e., $\beta$ ), they are prone to provide wrong numerical inversion results, which could prevent any subsequent quantitative characterization of specific areas in the scene. Besides the particular improvements proposed over an existing polarimetric inversion method, this paper is aimed at pointing out the necessity of checking quantitatively the accuracy of model-based PolSAR techniques for a reliable physical description of land covers beyond their proven utility for qualitative features extraction.
\end{abstract}

Keywords: model-based decomposition; polarimetric synthetic aperture radar (PolSAR); quantitative analysis; Monte Carlo simulations

\section{Introduction}

In the past few decades, many studies have shown that polarimetric decomposition is a useful technique to interpret physically the scattering mechanisms present in the scene when fully polarimetric synthetic aperture radar (PolSAR) data are available. A classical and a recent review of this methodology can be found in [1,2], respectively. The two most used types of incoherent PolSAR decomposition techniques are eigenvalue-eigenvector-based method and model-based methods [3]. The Freeman-Durden three-component decomposition and Yamaguchi four-component decomposition are the original works of model-based incoherent decomposition methods $[4,5]$. 
Based on these two methods, many improvements and advancements have been proposed [6-26]. Among them, a very active research topic is focused on a more general decomposition model and on the exploitation of the complete information in the covariance or coherency matrix [2]. A representative study of this category is the general polarimetric model-based decomposition method proposed recently by Chen et al. [22]. In this method, the decomposition model considers four different volume models previously proposed in the literature, a general surface scattering model involving orientation effect, a general double-bounce scattering model also involving orientation effect, and also a helix scattering model. This general approach can be used to obtain simultaneously all model parameters using a nonlinear least squares optimization technique. It overcomes the well-known limitations in traditional three-component and four-component decomposition methods such as model inversion priority, branch conditions, and negative powers. The experiments based on space-borne and airborne PolSAR data over urban areas demonstrated that this approach can efficiently improve the decomposition performance. Theoretically, this decomposition method is one of the most complete and general within the topic. However, most of the existing model-based decomposition studies are not focused on the retrieval of physical parameters (e.g., soil moisture or trunk dielectric constant) from the outputs of the decomposition, and they do not provide any other conclusion beyond the retrieved power of every scattering mechanism and its exploitation for target detection and land classification [27-36]. Thus far, very few studies have performed an in-depth analysis of the incoherent model-based decomposition concept (i.e., Freeman-Durden concept) for quantitative remote sensing applications. Some cases are the works by Jagdhüber et al. [37], Huang et al. [38], Di Martino et al. [39], and He et al. [40] focused on soil moisture inversion, which has actually been an active research line since some years ago. Nevertheless, the current state of the start on PolSAR decomposition techniques suggests that quantitative accuracy of parameters retrieved from model-based incoherent approaches is still an open issue. This statement is supported on the fact that all well-known model-based decompositions after Freeman-Durden have been designed to alleviate the initial limitations on the original approach. For such purpose, the strategy that has been initially followed has been constrained by the objective of finding a more reliable balance on the backscattering powers assigned to each mechanism. All these techniques are supported by physically-based models, which means that their outputs should be linked in a straightforward fashion to physical parameters such as dielectric constants or features of vegetation particles. Likewise, the backscattering powers of every scattering mechanism are reasonably expected to exhibit some correlation (depending on the scene) with biophysical parameters as it has been demonstrated in lots of both pioneering and current works by using the polarimetric channels as inputs and then by obtaining empirical or semi-empirical relationships to retrieve land cover features. Nevertheless, this assessment is lacking within the topic since the parameter accuracy has not been addressed so far in the way we propose in this study. The question we treat in this paper is how reliable are the retrieved backscattering powers from different scattering mechanisms, or backscattering ratios (i.e., $\alpha$ and $\beta$ ), or vegetation particle descriptors.

In this paper, we focus on this issue and a performance analysis of the whole parameter set is carried out on the basis that the approach proposed by Chen et al. in [22] is general enough for this purpose. This approach makes use of a nonlinear optimization procedure to retrieve model parameters, which is prone to yield non-physical solutions. Therefore, the objective is to check the consistency of these retrievals and propose modifications to improve the performance accuracy. These modifications include a redefinition of boundary conditions, a transformation of variables, and a different criterion for values initialization. In order to analyze the performance of the proposed improvements, a series of Monte Carlo simulation tests over different scattering cases are performed. A detailed comparative analysis of the results provided by both the proposed algorithm and Chen's one has been conducted. Finally, L-band E-SAR PolSAR images with ground measurements from the AgriSAR2006 campaign are employed for testing purposes as well.

The paper is organized as follows. In Section 2, the general polarimetric decomposition framework proposed by Chen et al. [22] is briefly reviewed. Section 3 describes the proposed modifications 
on the optimization algorithm employed for model parameter inversion. Section 4 presents a number of Monte Carlo simulation tests and the corresponding result analysis. Then, in Section 5, other decomposition models that are frequently used by PolSAR practitioners have been implemented and their outputs for L-band E-SAR data are compared to both Chen's results and the ones we obtained in this study. Further discussions are given in Section 6. Finally, conclusions of this work are drawn in Section 7.

\section{General Polarimetric Decomposition Model}

In this paper, the starting point is the general polarimetric decomposition model proposed by Chen et al. [22]. It includes four scattering components, i.e., volume scattering, surface scattering, double-bounce scattering, and helix scattering. More importantly, it actually exploits a number of advances and key ideas that were previously proposed in the literature.

\subsection{Volume Scattering Model}

In the research field of polarimetric decomposition, volume scattering from vegetation is often described as from randomly oriented dipoles with different probability density functions (pdf) with respect to orientation angle and particle anisotropy. For instance, in the classic Freeman-Durden three-component decomposition, the pdf is considered as a uniform distribution (named hereafter random dipoles model) [4]. Another two usual pdfs are sine and cosine distribution, first proposed in the Yamaguchi four-component decomposition method, and hence we can name them as horizontal and vertical dipoles models [5]. The general model of Chen includes these three classic volume scattering models, and it also considers another representative model, i.e., the highest entropy model proposed by An et al. [9,10]. The coherency matrix of the general volume scattering model can be expressed as [22]

$$
T_{v}=f_{v}\left[\begin{array}{ccc}
a & d & e \\
d^{*} & b & f \\
e^{*} & f^{*} & c
\end{array}\right],
$$

where the diagonal elements are real-valued and the others are complex-valued. Then, the four types of volume scattering models in Chen et al. [22] can be rewritten as

$$
\begin{gathered}
T_{\text {vol-random }}=\frac{1}{4} f_{v}\left[\begin{array}{lll}
2 & 0 & 0 \\
0 & 1 & 0 \\
0 & 0 & 1
\end{array}\right] T_{\text {vol-entropy }}=\frac{1}{3} f_{v}\left[\begin{array}{lll}
1 & 0 & 0 \\
0 & 1 & 0 \\
0 & 0 & 1
\end{array}\right], \\
T_{\text {vol-horizontal }}=\frac{1}{30} f_{v}\left[\begin{array}{ccc}
15 & 5 & 0 \\
5 & 7 & 0 \\
0 & 0 & 8
\end{array}\right] T_{\text {vol-vertical }}=\frac{1}{30} f_{v}\left[\begin{array}{ccc}
15 & -5 & 0 \\
-5 & 7 & 0 \\
0 & 0 & 8
\end{array}\right],
\end{gathered}
$$

In the inversion, the type of volume scattering model that best fits the data is selected, for which a residue minimization is applied as explained later in the text.

\subsection{Surface Scattering Model}

The basic surface scattering model adopted by Chen et al. [22] is the Bragg scattering model, which is also assumed in both Freeman-Durden and Yamaguchi decomposition models [4,5]. The Bragg scattering model, also known as Small Perturbation Model, is a special case of Integral Equation Method (IEM) model for slightly rough surfaces [41]. The coherency matrix of Bragg scattering model has the form $[41,42]$ 


$$
T_{s}=f_{s}\left[\begin{array}{ccc}
1 & \beta^{*} & 0 \\
\beta & |\beta|^{2} & 0 \\
0 & 0 & 0
\end{array}\right],
$$

where $\beta$ is theoretically a complex value. However, in the model inversion, we can ignore the imaginary part of $\beta(\beta \approx \operatorname{Re}(\beta))$ in most natural scenes for microwave regime, because its value is often a negligible number. The value of $\beta$ depends on horizontal $(\mathrm{H})$ and vertical $(\mathrm{V})$ Bragg reflection coefficients $\left(R_{H}, R_{V}\right)$ for horizontally and vertically polarized waves and it has the form

$$
\beta=\frac{R_{H}-R_{V}}{R_{H}+R_{V}},
$$

where these two coefficients depend on the local incidence angle $\theta$ and the relative dielectric constant of the surface $\varepsilon_{S}$. The expressions are written as [3]

$$
\begin{aligned}
& R_{H}=\frac{\cos \theta-\sqrt{\varepsilon_{S}-\sin ^{2} \theta}}{\cos \theta+\sqrt{\varepsilon_{S}-\sin ^{2} \theta}} \\
& R_{V}=\frac{\left(\varepsilon_{S}-1\right)\left(\sin ^{2} \theta-\varepsilon_{S}\left(1+\sin ^{2} \theta\right)\right)}{\left(\varepsilon_{S} \cos \theta+\sqrt{\varepsilon_{S}-\sin ^{2} \theta}\right)^{2}}
\end{aligned}
$$

Chen et al. [22] proposed considering the orientation effect by terrain slopes which induces a rotation along the radar line of sight on the coherency matrix [13,14]. The general form of rotation coherency matrix with a rotation angle $\psi$ is

$$
R_{3}(\psi)=\left[\begin{array}{ccc}
1 & 0 & 0 \\
0 & \cos 2 \psi & \sin 2 \psi \\
0 & -\sin 2 \psi & \cos 2 \psi
\end{array}\right],
$$

Therefore, the most general surface scattering model can be expressed as

$$
T_{S}\left(\psi_{S}\right)=R_{3}\left(\psi_{S}\right) T_{S} R_{3}^{H}\left(\psi_{S}\right),
$$

where $H$ denotes conjugate transposition operator.

\subsection{Double-Bounce Scattering Model}

The double-bounce scattering mechanism is modeled by dihedral scattering from two orthogonal reflection planes with same or different dielectric properties, such as ground-trunk for forest areas, ground-wall for urban areas, etc. [4]. In this case, the coherency matrix of double-bounce scattering model can be described mainly by Fresnel reflection coefficients and has the form [3]

$$
T_{\mathrm{d}}=f_{d}\left[\begin{array}{ccc}
|\alpha|^{2} & \alpha & 0 \\
\alpha^{*} & 1 & 0 \\
0 & 0 & 0
\end{array}\right]
$$

where $\alpha$ is a complex value which depends on the horizontal and vertical Fresnel reflection coefficients $R$ of the two reflection planes and on a phase difference between the HH and VV channels $\phi$ that may be caused by a differential propagation introduced by a vegetation layer if present. The expression is written as [3] 


$$
\alpha=\left(R_{T H} R_{S H}-e^{j \phi} R_{T V} R_{S V}\right) /\left(R_{T H} R_{S H}+e^{i \phi} R_{T V} R_{S V}\right)
$$

with

$$
\begin{gathered}
R_{i H}=\frac{\cos \theta_{i}-\sqrt{\varepsilon_{i}-\sin ^{2} \theta_{i}}}{\cos \theta_{i}+\sqrt{\varepsilon_{i}-\sin ^{2} \theta_{i}}} \\
R_{i V}=\frac{\varepsilon_{i} \cos \theta_{i}-\sqrt{\varepsilon_{i}-\sin ^{2} \theta_{i}}}{\varepsilon_{i} \cos \theta_{i}+\sqrt{\varepsilon_{i}-\sin ^{2} \theta_{i}}},
\end{gathered}
$$

where $i \in\{\mathrm{T}, S\}$ for vertical plane and surface plane, respectively. The local incidences for the two reflection planes are $\theta_{S}=\theta$ and $\theta_{\mathrm{T}}=\pi / 2-\theta_{S}$, respectively. Similarly, in the model of Chen, a possible orientation effect on the double-bounce scattering has also been considered and is modeled by a rotation matrix. The resulting general double-bounce scattering model can be written as

$$
T_{d}\left(\psi_{D}\right)=R_{3}\left(\psi_{\mathrm{D}}\right) T_{\mathrm{d}} R_{3}^{H}\left(\psi_{\mathrm{D}}\right)
$$

\subsection{Helix Scattering Model}

The helix scattering component proposed by Yamaguchi et al. is aimed at describing the reflection asymmetry case. The coherency matrix of helix scattering is roll-invariant and has the form [6]

$$
T_{c}=\frac{1}{2} f_{\mathcal{c}}\left[\begin{array}{ccc}
0 & 0 & 0 \\
0 & 1 & \pm j \\
0 & \mp j & 1
\end{array}\right]
$$

\section{Parameters Inversion}

\subsection{Inversion Model}

The general decomposition for the observed coherency matrix $T$ is given by [22]

$$
T=T_{v}+T_{s}\left(\psi_{S}\right)+T_{d}\left(\psi_{D}\right)+T_{c}+T_{\text {residual }}
$$

where $T_{\text {residual }}$ describes the difference between observations and models, including the errors from model mismatch and observation noise.

\subsection{Inversion Algorithm}

Because $T$ is a Hermitian matrix and the diagonal elements are real-valued, it can provide up to nine real independent observations. In the general decomposition model, the number of unknowns is also nine $\left(X=\left\{f_{v}, f_{s}, f_{d}, \operatorname{Re}(\alpha), \operatorname{Im}(\alpha), \beta, \psi_{S}, \psi_{D}\right\}\right)$. Chen et al. [22] adopted a nonlinear least squares optimization method for inverting this model, and the optimization criterion is the minimization of the sum of squares of residuals [22]

$$
\min :\left\|T_{\text {residual }}\right\|_{2}^{2}
$$

As it is usual in nonlinear optimization, one has to set initial values and boundary conditions for the optimization algorithm. In Chen et al. [22], the trust-region-reflective algorithm is used in the optimization $[43,44]$, the initial values correspond to the outputs from the traditional Yamaguchi four-component decomposition, and the boundary conditions are [22]

$$
\begin{gathered}
0 \leq f_{v}, f_{s}, f_{d} \leq S P A N 0 \leq f_{c} \leq 2\left|\operatorname{Im}\left(T_{23}\right)\right| \\
-\frac{\pi}{4} \leq \psi_{S}, \psi_{D} \leq \frac{\pi}{4} \quad|\beta|,|\alpha|<1
\end{gathered}
$$




\subsection{Modification of the Inversion Algorithm}

It is known that solutions in nonlinear optimization algorithms may fall to local minima which do not correspond to the global optimum or may correspond to physically non-feasible solutions. This can be due to several reasons, such as a poor selection of initial values, unfeasible boundary conditions, or problem overparameterization. Therefore, we propose some modifications to improve the optimization procedure used for inversion. They are grouped into two categories: modifications in the variables and a selection of better initial values.

\subsubsection{Modification in the Variables}

The ways we adopt to modify the variables considers two aspects: redefined boundary conditions, and transformation of variables, which are described next.

\section{Redefined Boundary Conditions}

The basic idea to redefine the boundary conditions is based on making use of some physical prior information and implicit conditions in the decomposition model itself. In the decomposition model, there are two parameters, i.e., $\alpha$ and $\beta$, which depend on relative dielectric constants and incidence angle. Besides, $\alpha$ depends on the phase difference $\phi$ whose theoretical range is $\left[-180^{\circ}, 180^{\circ}\right]$, and it will be zero for the random volume case. Therefore, for every specific incidence angle and a particular value of the phase difference, we can compute all the possible values of $\alpha$ in a 2-D space according to the model and the range of both relative dielectric constants. Then we can calculate its maximum and minimum value. Similarly, the possible values of $\beta$ in a 1-D space can be obtained using a range of soil dielectric constants and a specific incidence angle. Based on these computations, we can analyze the dependence of $\alpha$ and $\beta$ on these parameters according to the model and redefine their boundaries.

In our case, we adopt an empirical physical range of dielectric constant, i.e., $\varepsilon \in[2,41]$. This is not the largest feasible range for the dielectric constant in all cases, however, it covers a wide enough interval for soil moisture in most real scenarios [38,39,41,45]. In addition, such an interval (or a similar one, but never a much wider one) should be kept for many applications, not only for soil moisture retrieval, because any soil dielectric constant outside this range would correspond to a soil with non-physical properties. Figure 1 shows the maximum and minimum values of the magnitude $|\alpha|$ and the argument $\operatorname{Arg}(\alpha)$ assuming different polarimetric phase differences $\phi$ after selecting a range of incidence angles (e.g., 25 to 55 degrees corresponding to real data provide by E-SAR sensor) and the mentioned range of dielectric constants (2 to 41). There are two couples of incidence angles providing equal results, i.e., $\left(\theta=35^{\circ}, \theta=55^{\circ}\right)$ and $\left(\theta=40^{\circ}, \theta=50^{\circ}\right)$. From the graphs in Figure 1 , it is clear that $|\alpha|$ achieves the maximum values at $\phi= \pm 180^{\circ}$ and the minimum values at $\phi=0$, whereas $\operatorname{Arg}(\alpha)$ achieves the maximum value when $\phi$ is around $-90^{\circ}$ and the minimum value when $\phi$ is around $90^{\circ}$. In any case, the extreme values depend on the incidence angle. The maximum of the magnitude $|\alpha|$ can be greater than 1 , even when the phase difference is zero in the case of the steep incidence angles. Figure 2 shows a 2-D chart including all possible values of $|\alpha|$ at $\theta=25^{\circ}$ and $\phi=0$. As seen, at steep incidences the value of $|\alpha|$ is larger than 1 for all dielectric conditions of soil whenever the dielectric constant of trunk is low, i.e., a very dry trunk [46]. On the contrary, $\operatorname{Arg}(\alpha)$ exhibits both positive and negative values and the maximum and minimum values are always constrained in the $[-\pi / 2, \pi / 2]$ interval. It means that the complex parameter $\alpha$ will be always located into the first and fourth quadrant in the complex plane, i.e., the real part is always positive.

Based on these analyses, we found that at a fixed incidence angle, the boundaries of $|\alpha|$ and $\operatorname{Arg}(\alpha)$ depend strongly on the range of the polarimetric phase difference $\phi$. Nevertheless, as in a real case the value of $\phi$ is not known, we need to make some assumptions. To this aim, we resort to the physical interpretation of Pauli decomposition. As it is well-known, the $\mathrm{HH}+\mathrm{VV}$ and $\mathrm{HH}-\mathrm{VV}$ channels represent surface and horizontal double-bounce scattering mechanisms, respectively. Therefore, in the coherency matrix that corresponds to double-bounce scattering the T11 
element is less than the T22 element after assuming the power of $\mathrm{HH}+\mathrm{VV}$ must be less than that of $\mathrm{HH}-\mathrm{VV}$. Under this assumption, the corresponding boundary conditions for $\alpha$ are set as: $|\alpha|<1$. By doing so the range of the polarimetric phase difference $\phi$ will be automatically limited under the constraint of $\alpha$. However, we cannot directly define the boundary conditions of real and imaginary part of $\alpha$ from this assumption when we try to use the same unknown vector as Chen's method. Therefore, in order to embed this constraint into the parameter solving system (i.e., Equation (13)), the unknown parameters about $\alpha$ should be magnitude and argument instead of real and imaginary part. Then, in the nonlinear optimization system, the unknown vector should be transformed to $X=\left\{f_{v}, f_{s}, f_{d}, f_{c},|\alpha|, \operatorname{Arg}(\alpha), \beta, \psi_{s}, \psi_{D}\right\}$. Therefore, considering the model and this assumption, the redefined boundary conditions of $\alpha$ for any pixel $i$ should be:

$$
\begin{aligned}
& |\alpha|_{\min }\left\{\phi=0, \theta=\theta_{i}\right\}<|\alpha|<1 \\
& \operatorname{Arg}(\alpha)_{\min }\left\{\phi=90^{\circ}, \theta=\theta_{i}\right\}<\operatorname{Arg}(\alpha)<\operatorname{Arg}(\alpha)_{\max }\left\{\phi=-90^{\circ}, \theta=\theta_{i}\right\}
\end{aligned}
$$

where $|\alpha|_{\min }\left\{\phi=0, \theta=\theta_{i}\right\}$ denotes the minimum value of magnitude of $\alpha$ with zero polarimetric phase difference at the incidence angle of pixel $i . \operatorname{Arg}(\alpha)_{\min }\left\{\phi=90^{\circ}, \theta=\theta_{i}\right\}$ and $\operatorname{Arg}(\alpha)_{\max }\left\{\phi=-90^{\circ}, \theta=\theta_{i}\right\}$ represent the minimum and maximum value of argument of $\alpha$ with polarimetric phase difference of $\pm 90^{\circ}$ at the incidence angle of pixel $i$, respectively. Similarly, we show in Figure 3 the dependence of $\beta$ on the dielectric constant of soil $\varepsilon_{s}$ at different incidence angles. It can be seen that the values of $\beta$ decrease with the increase of $\varepsilon_{s}$ and all the values are negative. The domain of $\beta$ becomes wider from near-range (steep incidence angles) to far-range (shallow incidence angles). In the case of $\beta$, its boundaries can be determined directly by the model. Accordingly, the redefined boundary condition of $\beta$ for pixel $i$ is

$$
\beta_{\min }\left\{\theta=\theta_{i}\right\} \leq \beta \leq \beta_{\max }\left\{\theta=\theta_{i}\right\},
$$

where $\beta_{\min }\left\{\theta=\theta_{i}\right\}$ and $\beta_{\max }\left\{\theta=\theta_{i}\right\}$ represent the minimum and maximum values of $\beta$ at the incidence angle of pixel $i$, respectively.

The redefinition of upper and lower bounds for ground backscattering coefficients is done by considering some implicit conditions. For the polarimetric model-based decomposition methods, the power of surface scattering and double-bounce scattering should be always less than the span, i.e.,

$$
\begin{aligned}
& 0 \leq P_{S}=f_{s}\left(1+|\beta|^{2}\right) \leq S P A N \\
& 0 \leq P_{d}=f_{d}\left(1+|\alpha|^{2}\right) \leq S P A N
\end{aligned},
$$

Since the $\alpha$ and $\beta$ are also unknown parameters in equations, directly adding Equation (18) as constraints is like adding two additional inequality conditions inside the parameters inversion, hence obviously it would make the problem more complicated and maybe it would lead to other numerical issues. As a compromise solution, the redefined boundary conditions for both parameters can be derived as

$$
0 \leq f_{s} \leq \frac{S P A N}{\left(1+|\beta|_{\min }^{2}\right)} 0 \leq f_{d} \leq \frac{S P A N}{\left(1+|\alpha|_{\min }^{2}\right)},
$$

In summary, compared with the general boundary conditions employed in Chen et al. [22], in agreement with the scattering models, the redefined boundary conditions provide: (1) the lowest possible values of $|\alpha|$; (2) the largest and lowest possible values of $\operatorname{Arg}(\alpha)$; (3) the largest and lowest possible values of $\beta$; and (4) the largest possible values of $f_{s}, f_{d}$. Moreover, the boundary conditions change adaptively for different local incidence angles at pixel level. 
Variable Transformation

The second improvement consists of applying a variable transformation based on the boundary conditions and specific transformation functions. The basic idea of this transformation is to change this variable constrained problem to a variable unconstrained case after choosing a transformation function. The main advantage of this change is that any unconstrained optimization algorithm could be directly used to solve this problem [47] and the ranges of all the parameters would be the same, hence making the problem to be solved more easily [48]. In our case, all model parameters are dual-bounded, i.e., they exhibit lower and upper bounds. Therefore, we utilize an inverse tangent function to do the transformation. For a dual-bounded scalar or vector of variables $X$, the boundary conditions are $L B \leq X \leq U B$. Then the arc-tangent transformation is

$$
X=L B+(U B-L B) \times(\operatorname{atan}(U)+\pi / 2) / \pi,
$$

where the range of $\operatorname{atan}($.$) is [-\pi / 2, \pi / 2]$. Figure 4 shows a plot representing the relationship between the original variable and the new one after variable transformation. From this figure, it is obvious to see that the range of $X$ has not been changed and is also $[L B, U B]$; however, the new variables $U$ are unbounded. Then, by applying this transformation to the optimization object function, the optimization problem becomes an unconstraint optimization depending on the vector of variables $U$.

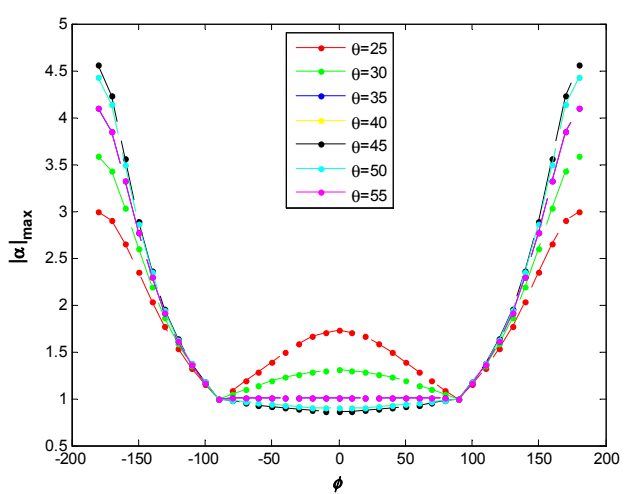

(a)

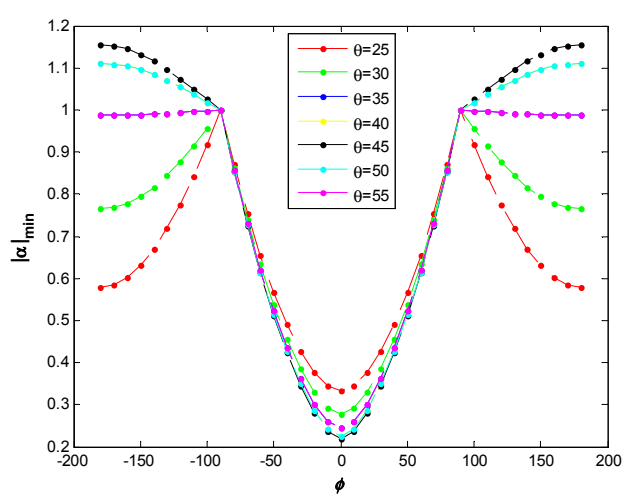

(c)

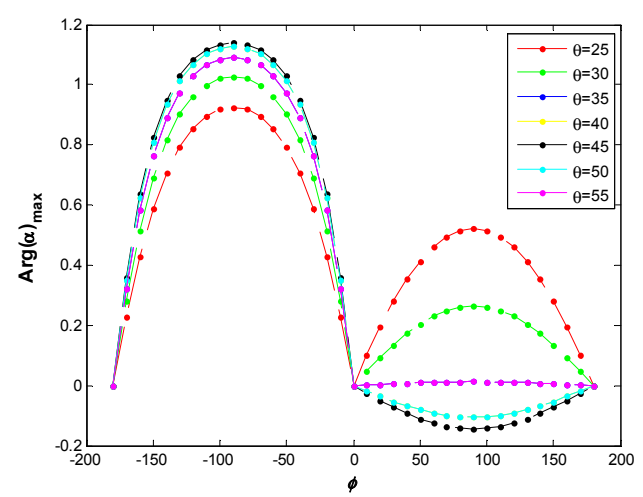

(b)

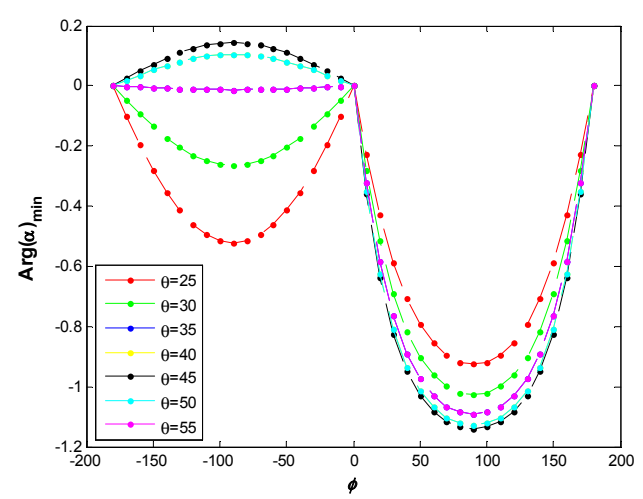

(d)

Figure 1. Dependence of magnitude and argument of $\alpha$ on polarimetric phase difference $\phi$ at different incidence angles: (a) maximum of magnitude; (b) maximum of argument; (c) minimum of magnitude; and (d) minimum of argument. $\varepsilon_{\mathrm{S}}=\varepsilon_{t} \in[2,41], \phi \in[-180,180], \theta \in[25,55]$. 


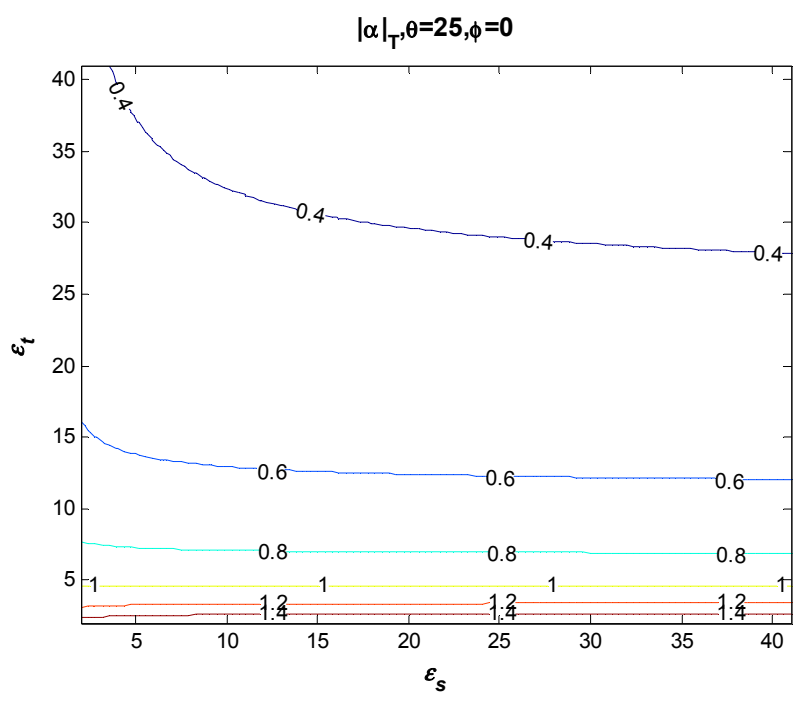

Figure 2. Dependence of absolute value of $\alpha$ on the dielectric constant of soil and trunk when incidence angle is 25 degrees and with zero polarimetric phase difference. $\varepsilon_{\mathrm{S}}=\varepsilon_{t} \in[2,41]$.

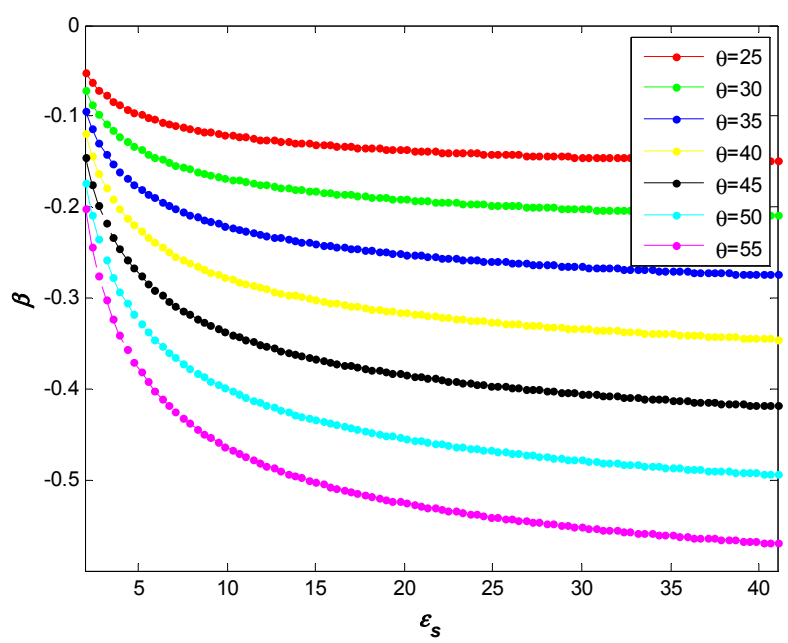

Figure 3. Dependence of $\beta$ on the soil dielectric constant at different incidence angles. $\varepsilon_{\mathrm{s}} \in[2,41]$, $\theta \in[25,55]$.

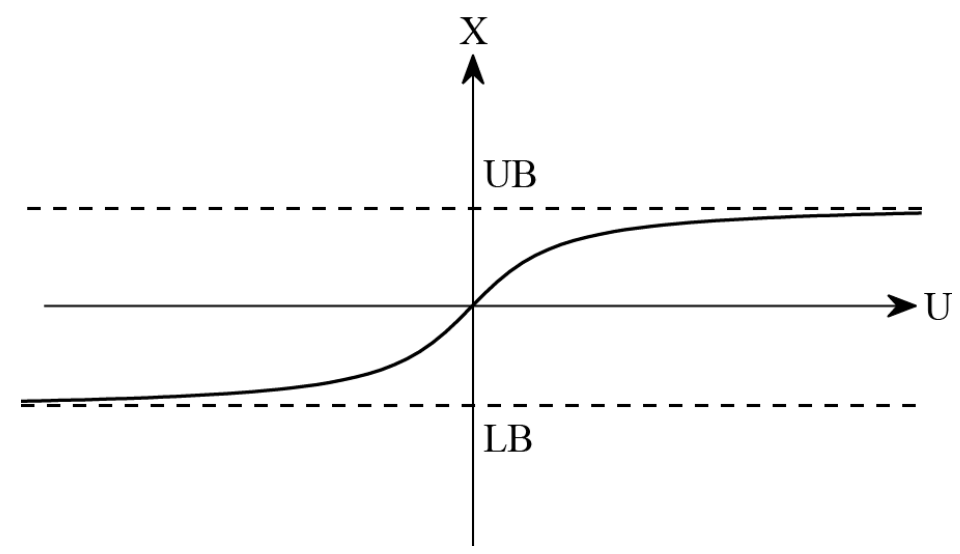

Figure 4. Relationship between an original variable $X$ and the new variable $U$ after variable transformation. 


\subsubsection{Modification for Initial Values}

Besides the aspects treated above, initial values are also an important aspect with large impact in nonlinear optimizations. The optimization in Chen et al. [22], makes use of initial values taken directly from the output of the conventional decomposition methods, such as Yamaguchi decomposition. In our tests, we found that in some cases these initial values are beyond the physical ranges we defined before. Hence, this strategy leads directly to unacceptable results. The reason is derived from the last step of Freeman-Durden or Yamaguchi decomposition. It is indeed an undetermined problem since there are four unknowns and three equations [21] as follows

$$
\left\{\begin{array}{l}
f_{s}+f_{d}|\alpha|^{2}=S \\
f_{s}|\beta|^{2}+f_{d}=D, \\
f_{s} \beta^{*}+f_{d} \alpha=C
\end{array}\right.
$$

The traditional way to solve this problem is to fix $\alpha$ or $\beta$ according to the relative higher contribution of either surface or double-bounce scattering. This strategy is valid whenever the target matches this assumption but it fails when there exists a mixed scattering process which is also a common behavior in many scenarios. Therefore, from the viewpoint of quantitative analysis, these are not actual solutions of model parameters and will lead to wrong results.

An alternative and simple way to set the initial values for $\alpha$ and $\beta$ consists in using just the mean value within their physically feasible ranges. Then, we go back to equations to solve $f_{s}$ and $f_{d}$ using a least squares method, because now the parameters solving equation system becomes an overdetermined problem with more equations than unknowns. In summary, our method to set initial values of parameters is

- $\quad f_{\mathrm{V}}, f_{\mathrm{c}}$ from Yamaguchi decomposition method

- $\alpha, \beta$ from the center of physical ranges

- $f_{s}, f_{d}$ from the least squares estimation based on the initial values of $\alpha, \beta$

- $\psi_{S}, \psi_{D}$ from the negative value of the polarization orientation angle derived by Lee et al. [13].

\section{Simulation Tests}

\subsection{Monte Carlo Simulation for PolSAR Data}

For simulating PolSAR data, the algorithm proposed in [3] can be used. The basic idea of this algorithm is to use a Gaussian random number generator to generate a complex Gaussian distribution random vector with zero mean and identity covariance matrix, and then to simulate a multi-look averaged covariance or coherence matrix based on a given covariance matrix. For every realization, the basic procedure is shown below [3]:

(1) Eigenvalue decomposition for the given covariance matrix $C$ and computation of the matrix $C^{1 / 2}$

$$
\begin{aligned}
& V^{H} C V=D \\
& C^{1 / 2}=V \times \sqrt{D}, \text { where } C^{1 / 2} \times\left(C^{1 / 2}\right)^{H}=C^{\prime}
\end{aligned},
$$

(2) Simulate a normal complex Gaussian random vector $v$ using Gaussian random number generators.

(3) Compute the simulated single look complex vector $u$ :

$$
u=C^{1 / 2} v,
$$

(4) Compute the n-looks averaged covariance matrix $C_{s i m}$ : 


$$
C_{\text {sim }}=\frac{1}{n} \sum_{1}^{n} u u^{H},
$$

(5) The corresponding coherency matrix is obtained by a special unitary transformation matrix. Alternatively, the coherency matrix simulation could be directly used from the beginning of the simulation, i.e., without conversion to covariance matrix.

\subsection{Results and Analysis}

We have synthesized covariance matrices according to the model described above and then we have obtained simulated data sets for testing purposes. We have simulated three typical cases to test our algorithm: (a) Case 1, without dominant component; (b) Case 2, with dominant surface scattering component; and (c) Case 3, with dominant double-bounce component. In our tests, we have adjusted the relationship between $f_{d}$ and $f_{s}$ to generate simulated measurements of different cases and for simplification, the volume model we adopted is $T_{\text {vol-random }}$ defined in Equation (2). The input model parameters used for the three cases are shown in Table 1, respectively. In every case, we performed 1000 realizations and the number of looks for reducing speckle noise is $15 \times 15$. Then we obtained 1000 samples of the multi-looked coherency matrix to test the algorithms. As we have proposed three modifications, we have analyzed first each single modification separately from the others in order to understand the individual contribution to the final results. Then, the combination of all of them is also analyzed. The histograms of the outputs provided by the different versions of the inversion approach are shown in Figures 4-6. In all cases, the results obtained with the original algorithm proposed by Chen et al. [22] are also used for comparison.

Table 1. Values for input parameters in three typical cases.

\begin{tabular}{|c|c|c|c|c|c|c|c|c|c|c|c|c|}
\hline & $f_{v}$ & $f_{s}$ & $f_{d}$ & $f_{c}$ & $\psi_{S}$ & $\psi_{D}$ & $\alpha$ & $\beta$ & $\theta$ & $\phi$ & $\varepsilon_{S}$ & $\varepsilon_{T}$ \\
\hline Case 1 & 5 & 5 & 5 & & & & & & & & & \\
\hline Case 2 & 5 & 5 & 2.5 & 0.01 & $-10^{\circ}$ & $-15^{\circ}$ & $0.3515-0.0768 \mathrm{i}$ & -0.3377 & $45^{\circ}$ & $10^{\circ}$ & 10 & 30 \\
\hline Case 3 & 5 & 2.5 & 5 & & & & & & & & & \\
\hline
\end{tabular}

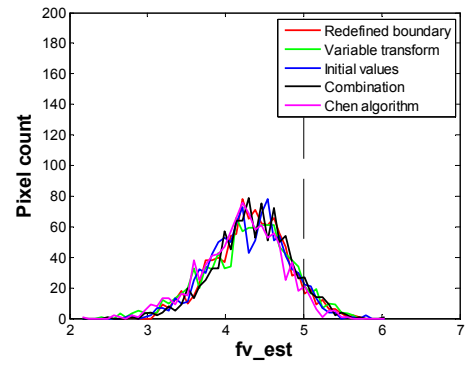

(a)

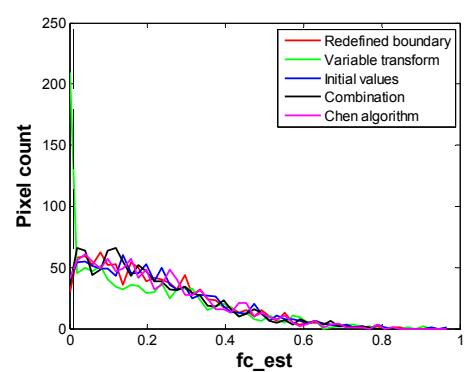

(d)

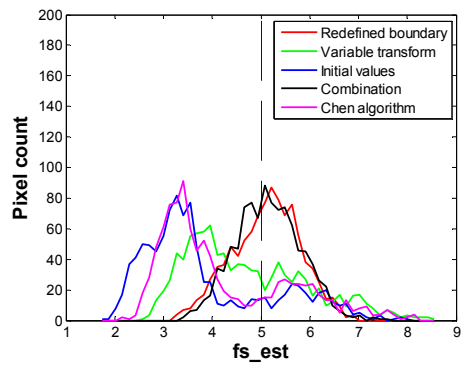

(b)

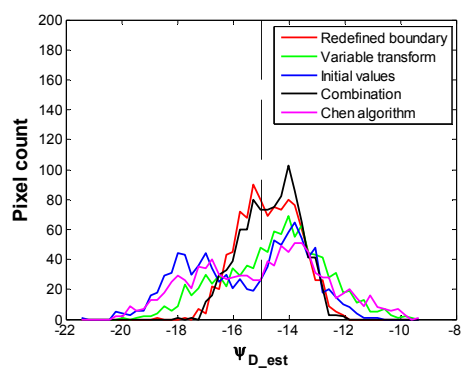

(e)

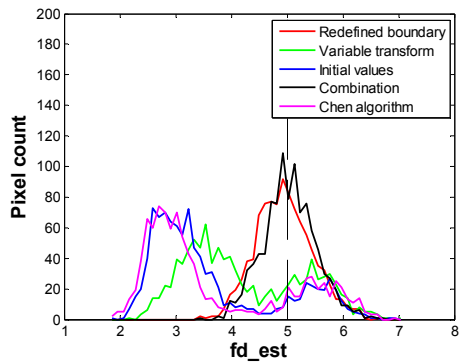

(c)

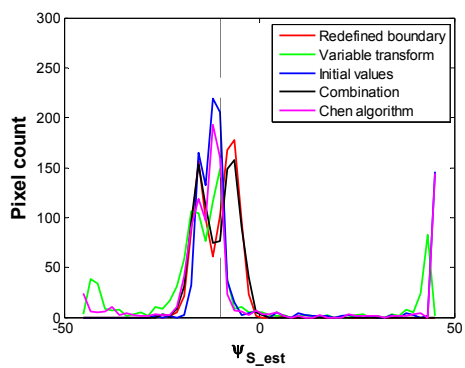

(f)

Figure 5. Cont. 


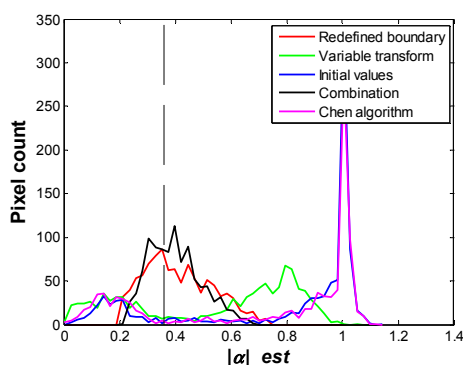

(g)

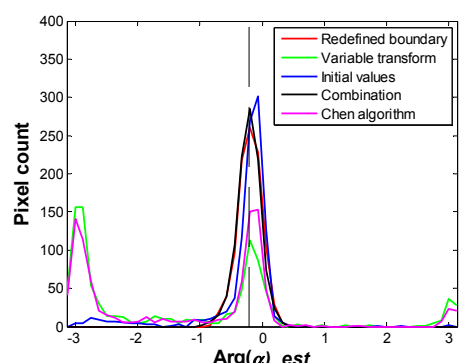

(h)

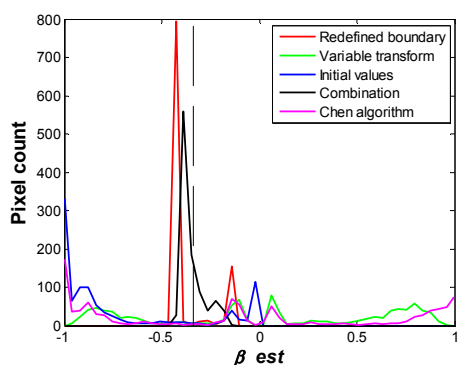

(i)

Figure 5. The histograms of inversion result of 9 parameters with different algorithms in Case 1: (a) Volume scattering coefficient; (b) Surface scattering coefficient; (c) Double-bounce scattering coefficient; (d) Helix scattering coefficient; (e) Rotation angle in surface scattering model; (f) Rotation angle in double-bounce scattering model; (g) Absolute value of double-bounce model parameter $\alpha$; (h) Argument of double-bounce model parameter $\alpha$; and (i) Surface scattering model parameter $\beta$. The vertical dashed line denotes the true value.

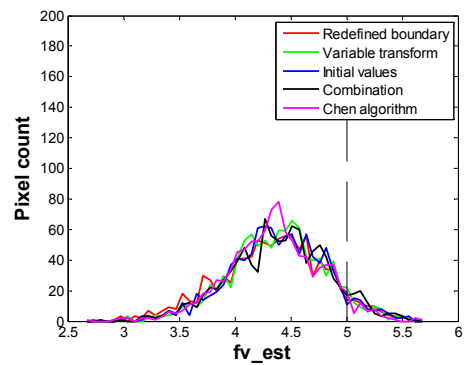

(a)

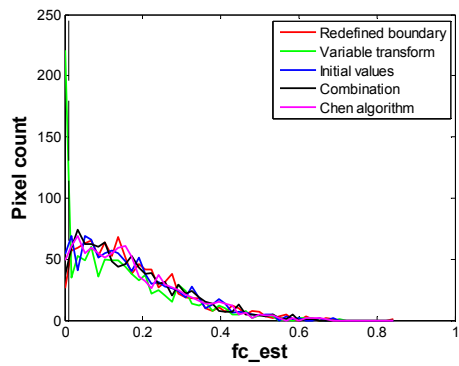

(d)

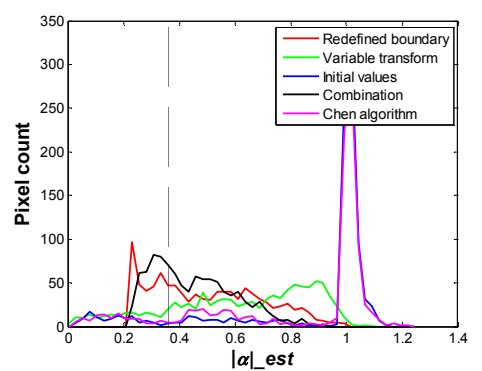

(g)

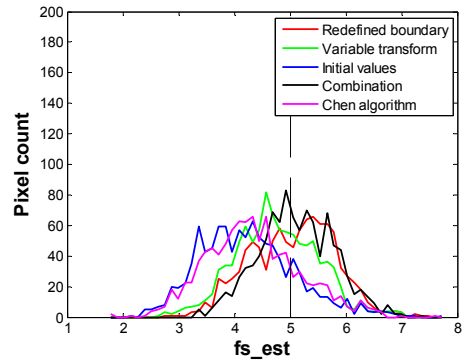

(b)

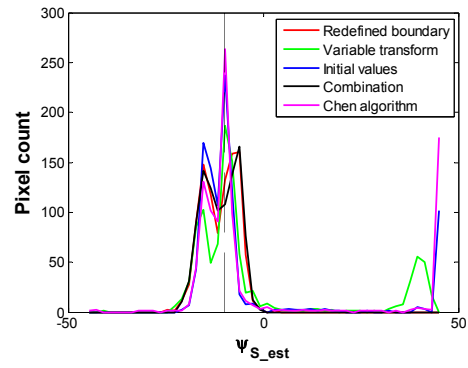

(e)

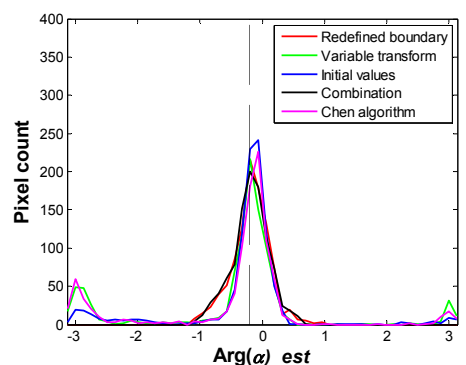

(h)

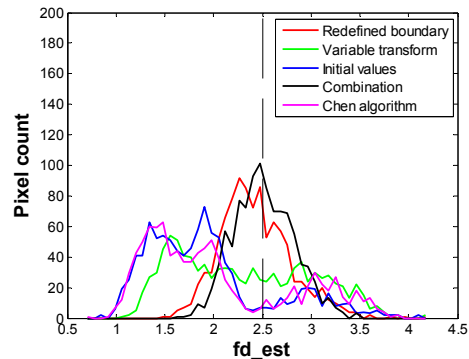

(c)

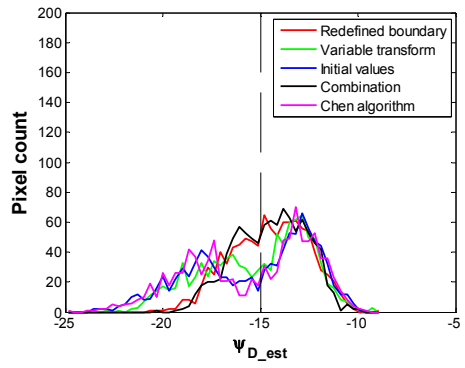

(f)

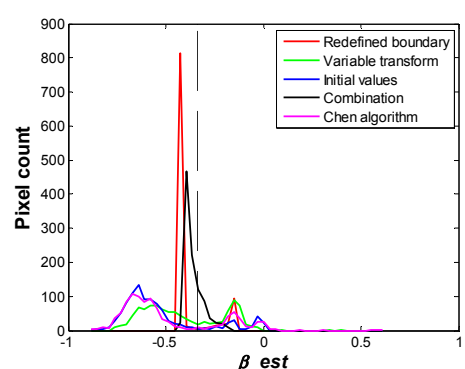

(i)

Figure 6. The histograms of inversion result of 9 parameters with different algorithms in Case 2: (a) Volume scattering coefficient; (b) Surface scattering coefficient; (c) Double-bounce scattering coefficient; (d) Helix scattering coefficient; (e) Rotation angle in surface scattering model; (f) Rotation angle in double-bounce scattering model; (g) Absolute value of double-bounce model parameter $\alpha$; (h) Argument of double-bounce model parameter $\alpha$; and (i) Surface scattering model parameter $\beta$. The vertical dashed line denotes the true value. 
As shown in Figures 5-7, the different strategies are labeled with solid lines in different colors: redefined boundary in red, variable transform in green, initial values in blue, combination in black and Chen algorithm in pink. From the histograms, we can see that the inversion of the nine model parameters with the combination of all proposed strategies, as well as with just the redefined boundary strategy, show more robustness in a noisy scenario than the rest of strategies, including the original Chen's algorithm. Moreover, the combination of all proposed strategies exhibits the highest accuracy.

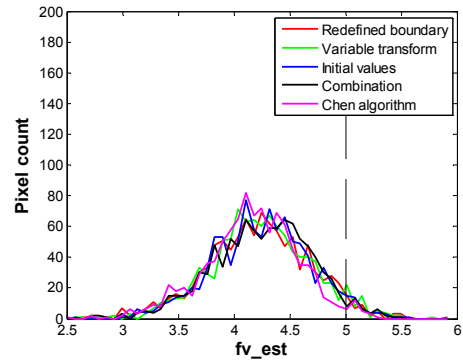

(a)

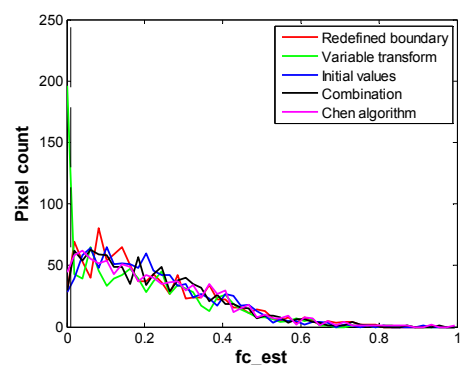

(d)

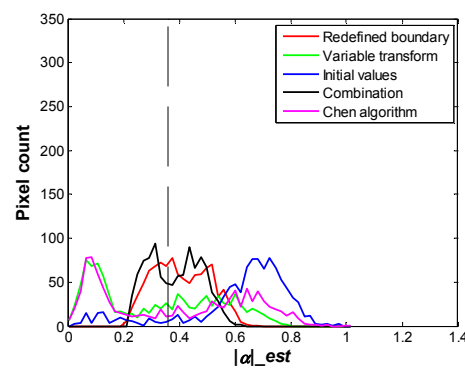

(g)

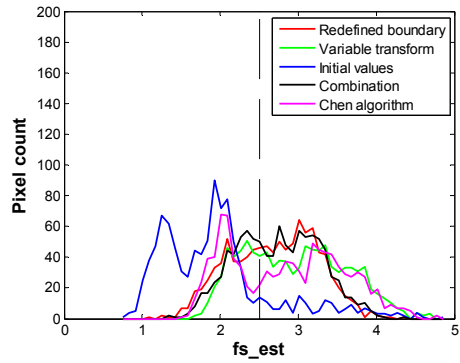

(b)

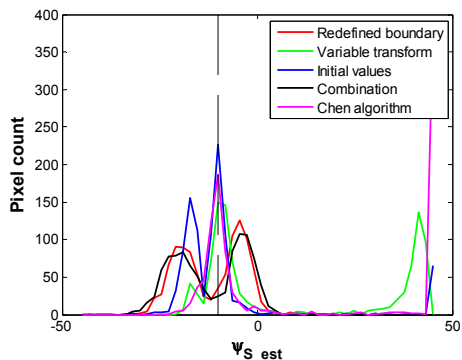

(e)

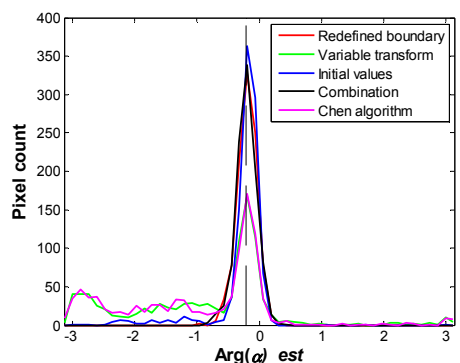

(h)

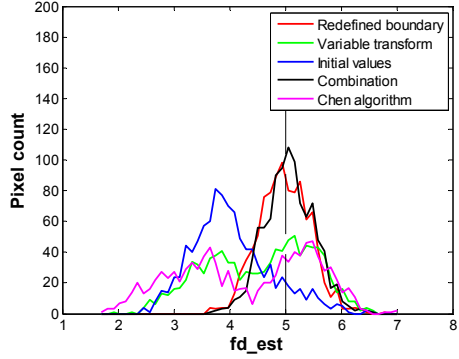

(c)

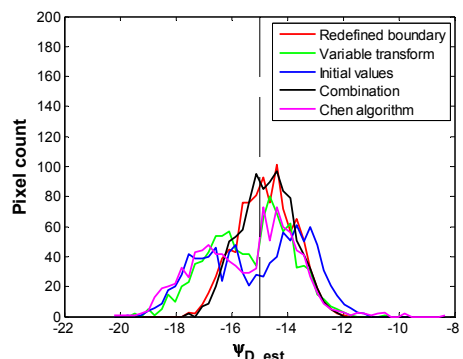

$(\mathbf{f})$

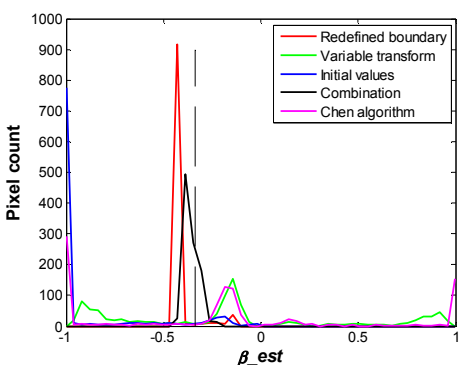

(i)

Figure 7. The histograms of inversion result of 9 parameters with different algorithms in Case 3: (a) Volume scattering coefficient; (b) Surface scattering coefficient; (c) Double-bounce scattering coefficient; (d) Helix scattering coefficient; (e) Rotation angle in surface scattering model; (f) Rotation angle in double-bounce scattering model; (g) Absolute value of double-bounce model parameter $\alpha$; (h) Argument of double-bounce model parameter $\alpha$; and (i) Surface scattering model parameter $\beta$. The vertical dashed line denotes the true value.

In order to further analyze the inversion performance of different model parameters, we divide them into three groups: (1) backscattering coefficients: $f_{v}, f_{s}, f_{d}, f_{c} ;(2)$ parameters related to dielectric constants for quantitative applications: $\alpha, \beta$; and (3) parameters related to rotation angles: $\psi_{S}, \psi_{D}$.

For all three cases, in the result of Chen's algorithm, the histograms of backscattering coefficient parameters for double-bounce and surface reveal a bias from the actual values, whereas the histograms of other parameters seem weird and more fluctuant and many of the solutions are located on the boundaries, such as $\operatorname{Arg}(\alpha)$ in Case 1 and Case 2, and $\beta$ in all three cases. It suggests that when employing Chen's algorithm in qualitative applications, the result is acceptable, whereas it is unreliable to use the inversion results in quantitative applications. On the contrary, the histograms of the results 
obtained by the proposed algorithm indicate that the estimation error is low enough for a reliable quantitative analysis based on the whole set of parameters. As for the inversion of the two rotation angle parameters, the results of all algorithms show that the double-bounce orientation angle $\psi_{D}$ is better estimated than the surface orientation angle $\psi_{S}$ in all three cases. These results show consistency with the results tested in build-up areas by using Chen's algorithm in [22] .In addition, compared with Chen's algorithm, the combination strategy achieves a noticeable improvement in the retrieval of both angles.

We have also calculated the bias and RMSE (Root Mean Square Error) of the inverted parameters with all different strategies as shown in Tables 2-4. These two statistic indexes show that:

(1) On average, all individual modification strategies are valid and in general they improve the parameter inversion accuracy with respect to the results of Chen's algorithm.

(2) The proposed algorithm can provide the best result and the redefinition of boundaries contributes more to the final result than other modifications.

(3) The algorithm is robust enough for different simulated scenarios considering different contributions for the main three scattering mechanisms.

Table 2. Bias and RMSE of different strategies in case 1.

\begin{tabular}{|c|c|c|c|c|c|c|c|c|c|c|}
\hline & \multicolumn{2}{|c|}{$\begin{array}{l}\text { Redefined } \\
\text { Boundary }\end{array}$} & \multicolumn{2}{|c|}{$\begin{array}{l}\text { Variable } \\
\text { Transform }\end{array}$} & \multicolumn{2}{|c|}{ Initial Values } & \multicolumn{2}{|c|}{ Combination } & \multicolumn{2}{|c|}{ Chen Algorithm } \\
\hline & $\begin{array}{c}\text { Mean } \\
\text { Bias }\end{array}$ & RMSE & $\begin{array}{c}\text { Mean } \\
\text { Bias }\end{array}$ & RMSE & $\begin{array}{c}\text { Mean } \\
\text { Bias }\end{array}$ & RMSE & $\begin{array}{c}\text { Mean } \\
\text { Bias }\end{array}$ & RMSE & $\begin{array}{c}\text { Mean } \\
\text { Bias }\end{array}$ & RMSE \\
\hline$f_{v_{-} \text {est }}$ & 0.7098 & 0.8275 & 0.7455 & 0.8926 & 0.7294 & 0.8538 & 0.6848 & 0.8069 & 0.8059 & 0.9364 \\
\hline$f_{S_{-}}$est & 0.5810 & 0.7129 & 1.0825 & 1.2653 & 1.6000 & 1.7485 & 0.5531 & 0.6896 & 1.3885 & 1.5279 \\
\hline$f_{d \_s s t}$ & 0.4088 & 0.5119 & 1.1306 & 1.2997 & 1.6198 & 1.7766 & 0.3728 & 0.4752 & 1.6500 & 1.8344 \\
\hline$f_{\text {c_est }}$ & 0.2079 & 0.2642 & 0.1792 & 0.2513 & 0.2120 & 0.2700 & 0.1977 & 0.2541 & 0.2036 & 0.2595 \\
\hline$\psi_{S \_ \text {est }}$ & 0.0688 & 0.0803 & 0.2422 & 0.3839 & 0.1980 & 0.3844 & 0.0741 & 0.0854 & 0.2384 & 0.4093 \\
\hline$\psi_{D \_e s t}$ & 0.0158 & 0.0192 & 0.0269 & 0.0326 & 0.0312 & 0.0367 & 0.0158 & 0.0189 & 0.0329 & 0.0392 \\
\hline$|\alpha| \_e s t$ & 0.1046 & 0.1328 & 0.3127 & 0.3449 & 0.4935 & 0.5353 & 0.0797 & 0.1018 & 0.4647 & 0.5117 \\
\hline $\operatorname{Arg}(\alpha) \_e s t$ & 0.1521 & 0.1926 & 1.7071 & 2.1068 & 0.3015 & 0.6247 & 0.1462 & 0.1894 & 1.4241 & 1.9032 \\
\hline B_est & 0.0979 & 0.1064 & 0.6186 & 0.7166 & 0.4950 & 0.5266 & 0.0519 & 0.0617 & 0.6829 & 0.7861 \\
\hline Avg_Mean & \multicolumn{2}{|c|}{0.2607} & \multicolumn{2}{|c|}{0.6717} & \multicolumn{2}{|c|}{0.6312} & \multicolumn{2}{|c|}{0.2418} & \multicolumn{2}{|c|}{0.7657} \\
\hline Avg_RMSE & \multicolumn{2}{|c|}{0.3164} & \multicolumn{2}{|c|}{0.8104} & \multicolumn{2}{|c|}{0.7507} & \multicolumn{2}{|c|}{0.2981} & \multicolumn{2}{|c|}{0.9120} \\
\hline
\end{tabular}

Table 3. Bias and RMSE of different strategies in case 2.

\begin{tabular}{|c|c|c|c|c|c|c|c|c|c|c|}
\hline & \multicolumn{2}{|c|}{$\begin{array}{l}\text { Redefined } \\
\text { Boundary }\end{array}$} & \multicolumn{2}{|c|}{$\begin{array}{l}\text { Variable } \\
\text { Transform }\end{array}$} & \multicolumn{2}{|c|}{ Initial Values } & \multicolumn{2}{|c|}{ Combination } & \multicolumn{2}{|c|}{ Chen Algorithm } \\
\hline & $\begin{array}{c}\text { Mean } \\
\text { Bias }\end{array}$ & RMSE & $\begin{array}{l}\text { Mean } \\
\text { Bias }\end{array}$ & RMSE & $\begin{array}{l}\text { Mean } \\
\text { Bias }\end{array}$ & RMSE & $\begin{array}{c}\text { Mean } \\
\text { Bias }\end{array}$ & RMSE & $\begin{array}{c}\text { Mean } \\
\text { Bias }\end{array}$ & RMSE \\
\hline$f_{V_{\text {V_est }}}$ & 0.6906 & 0.8135 & 0.6408 & 0.7474 & 0.6389 & 0.7460 & 0.6346 & 0.7488 & 0.6681 & 0.7717 \\
\hline$f_{s_{-} e s t}$ & 0.6267 & 0.7600 & 0.6301 & 0.7791 & 0.9768 & 1.1526 & 0.5477 & 0.6829 & 0.8993 & 1.0719 \\
\hline$f_{d \_e s t}$ & 0.2987 & 0.3736 & 0.6181 & 0.7087 & 0.7783 & 0.8539 & 0.2464 & 0.3071 & 0.8058 & 0.8779 \\
\hline$f_{\text {C_est }}$ & 0.1648 & 0.2111 & 0.1338 & 0.1872 & 0.1595 & 0.2062 & 0.1584 & 0.2035 & 0.1592 & 0.2056 \\
\hline$\psi_{S \_ \text {est }}$ & 0.0663 & 0.0785 & 0.2083 & 0.3719 & 0.1567 & 0.3276 & 0.0673 & 0.0784 & 0.2275 & 0.4232 \\
\hline$\psi_{D}$ est & 0.0295 & 0.0353 & 0.0396 & 0.0465 & 0.0461 & 0.0537 & 0.0277 & 0.033 & 0.0475 & 0.0551 \\
\hline$|\alpha| \_$est & 0.1906 & 0.2433 & 0.3169 & 0.3663 & 0.5499 & 0.5856 & 0.1332 & 0.1747 & 0.5117 & 0.5591 \\
\hline $\operatorname{Arg}(\alpha) \_e s t$ & 0.2351 & 0.3112 & 0.7519 & 1.3160 & 0.4842 & 0.9534 & 0.2324 & 0.3029 & 0.7271 & 1.2781 \\
\hline B_est & 0.0924 & 0.0988 & 0.1936 & 0.2149 & 0.2685 & 0.2843 & 0.0456 & 0.0523 & 0.2717 & 0.2915 \\
\hline Avg_Mean & \multicolumn{2}{|c|}{0.2661} & \multicolumn{2}{|c|}{0.3926} & \multicolumn{2}{|c|}{0.4510} & \multicolumn{2}{|c|}{0.2326} & \multicolumn{2}{|c|}{0.4798} \\
\hline Avg_RMSE & \multicolumn{2}{|c|}{0.3250} & \multicolumn{2}{|c|}{0.5264} & \multicolumn{2}{|c|}{0.5737} & \multicolumn{2}{|c|}{0.2871} & \multicolumn{2}{|c|}{0.6149} \\
\hline
\end{tabular}


Table 4. Bias and RMSE of different strategies in case 3.

\begin{tabular}{|c|c|c|c|c|c|c|c|c|c|c|}
\hline & \multicolumn{2}{|c|}{$\begin{array}{l}\text { Redefined } \\
\text { Boundary }\end{array}$} & \multicolumn{2}{|c|}{$\begin{array}{l}\text { Variable } \\
\text { Transform }\end{array}$} & \multicolumn{2}{|c|}{ Initial Values } & \multicolumn{2}{|c|}{ Combination } & \multicolumn{2}{|c|}{ Chen Algorithm } \\
\hline & $\begin{array}{c}\text { Mean } \\
\text { Bias }\end{array}$ & RMSE & $\begin{array}{c}\text { Mean } \\
\text { Bias }\end{array}$ & RMSE & $\begin{array}{c}\text { Mean } \\
\text { Bias }\end{array}$ & RMSE & $\begin{array}{c}\text { Mean } \\
\text { Bias }\end{array}$ & RMSE & $\begin{array}{c}\text { Mean } \\
\text { Bias }\end{array}$ & RMSE \\
\hline$f_{v \_e s t}$ & 0.7918 & 0.9048 & 0.7749 & 0.8807 & 0.7642 & 0.8686 & 0.7602 & 0.8705 & 0.8432 & 0.9374 \\
\hline$f_{\text {S_est }}$ & 0.4959 & 0.5901 & 0.6363 & 0.7938 & 0.806 & 0.9114 & 0.4806 & 0.5829 & 0.6753 & 0.7995 \\
\hline$f_{d \_e s t}$ & 0.3702 & 0.4631 & 0.8417 & 1.0466 & 1.0689 & 1.2076 & 0.3572 & 0.4513 & 1.112 & 1.3721 \\
\hline$f_{\text {C_est }}$ & 0.2088 & 0.2686 & 0.1758 & 0.2397 & 0.2064 & 0.2567 & 0.2072 & 0.2624 & 0.2137 & 0.2743 \\
\hline$\psi_{S}$ est & 0.1337 & 0.1536 & 0.3726 & 0.5464 & 0.1431 & 0.2777 & 0.1451 & 0.1621 & 0.4234 & 0.6151 \\
\hline$\psi_{D}$ est & 0.0149 & 0.0182 & 0.0215 & 0.0261 & 0.0276 & 0.0316 & 0.0142 & 0.0174 & 0.0254 & 0.0312 \\
\hline$|\alpha| \_$est & 0.0906 & 0.1111 & 0.1978 & 0.2221 & 0.3004 & 0.3205 & 0.0831 & 0.0962 & 0.2418 & 0.2652 \\
\hline $\operatorname{Arg}(\alpha) \_$est & 0.1260 & 0.1612 & 0.9457 & 1.3741 & 0.2045 & 0.4336 & 0.1289 & 0.1677 & 1.0018 & 1.4308 \\
\hline B_est & 0.0847 & 0.0878 & 0.4705 & 0.5976 & 0.564 & 0.5979 & 0.0379 & 0.0436 & 0.5479 & 0.6877 \\
\hline Avg_Mean & \multicolumn{2}{|c|}{0.2574} & \multicolumn{2}{|c|}{0.4930} & \multicolumn{2}{|c|}{0.4539} & \multicolumn{2}{|c|}{0.2460} & \multicolumn{2}{|c|}{0.5649} \\
\hline Avg_RMSE & \multicolumn{2}{|c|}{0.3065} & \multicolumn{2}{|c|}{0.6363} & \multicolumn{2}{|c|}{0.5451} & \multicolumn{2}{|c|}{0.2949} & \multicolumn{2}{|c|}{0.7126} \\
\hline
\end{tabular}

Assessment of the Residual

Both Chen's and the proposed method use residual minimization as a measure for optimal parameter solving. For further comparison, we have employed a normalized version of the residual as a quality index [22]

$$
R_{\min }=\left\|T_{\text {residual }}\right\|_{2}^{2} /\|T\|_{2}^{2}
$$

Because the coherency matrix is a Hermitian matrix, only the upper triangular elements participate in the optimization calculation. Then, the L2-norm is computed accordingly. The mean values of $R_{\text {min }}$ for Chen's method are $1.55 \times 10^{-4}, 1.96 \times 10^{-4}$ and $1.64 \times 10^{-4}$ for the three cases, whereas those for proposed combination method are a little larger $\left(1.89 \times 10^{-4}, 2.32 \times 10^{-4}\right.$ and $1.89 \times 10^{-4}$, respectively). The main reason for that comes from the boundary conditions in Chen's method, which are more relaxed and thus it leads to smaller residuals in the nonlinear optimization processing. However, in order to achieve this, the solutions of the unknowns will absorb larger errors with respect to the true values.

\section{Test with Real Data: AgriSAR2006 Campaign}

\subsection{Data Description}

The airborne PolSAR dataset we have tested was acquired during the ESA-funded AgriSAR2006 campaign, which involved 16 European institutions during a four month period in 2006. The test site is located in Western Pomerania in Northeast Germany, which is well established for simultaneous measurements of both airborne and in situ data. The detailed descriptions about the measurement accuracy and data processing can be found in [49]. Besides some forest and building areas, there are a variety of different crop types, including maize, sugar beet, winter wheat, winter barley and winter rape. Figure 8 shows the land cover map of the test site. In this campaign, full-polarimetric L-band images were acquired by the DLR E-SAR system with an incidence range of $25^{\circ}-55^{\circ}$. For this study we have employed an image acquired on the first date of the campaign where some of the fields corresponded to bare rough surfaces and others were in the early stages of development of crops with plants up to 19 centimeters high at most. The acquired date of this image is 19 April 2006 and the resolution is $2 \mathrm{~m}$ in the range direction and $1.2 \mathrm{~m}$ in the azimuth direction. 


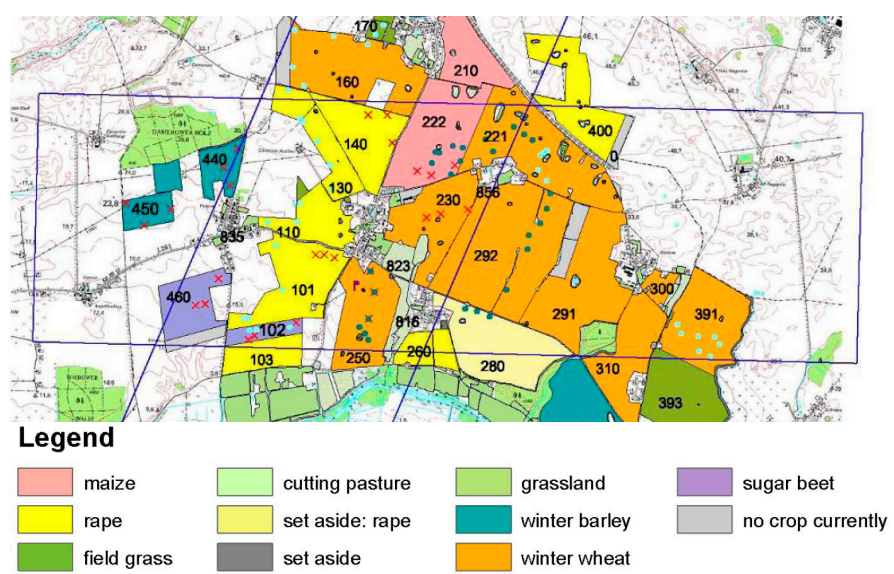

Figure 8. Land cover map with location of fields. The flight direction is from east to west (i.e., left to right in this picture). Near range is on the top and far range on the bottom.

\subsection{Results and Analysis}

Figure 9 shows the photographs of all crops types at the date of the radar observation. In addition to the farmland area, two forest areas (one in near-range, the other in far-range) and one building area are also selected to test the decomposition performance. In order to assess the performance, we have also obtained the solutions from a number of traditional incoherent model-based decomposition methods, namely: Y4O (Yamaguchi four-component decomposition, Y4O [5]), Y4R (Yamaguchi four-component decomposition with rotation, Y4R [15]), S4R (Yamaguchi four-component decomposition with rotation plus dihedral volume scattering model, S4R [18]), G4U (General four-component decomposition with unitary transformation, G4U [21]). A spatial multilook with a $9 \times 9$ boxcar filter to reduce speckle noise was applied. The conventional RGB composites obtained from the six decomposition methods are shown in Figure 10, whereas a comparative analysis of the results for each crop type is presented next.

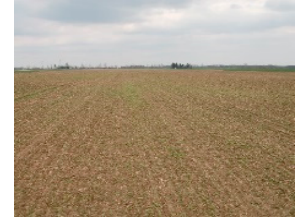

(a)

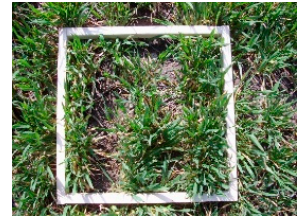

(b)

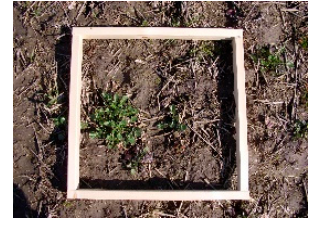

(c)

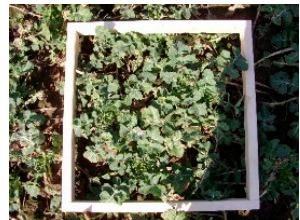

(d)

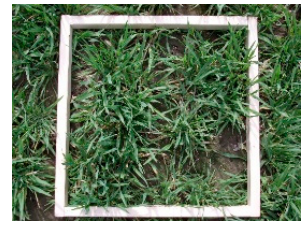

(e)

Figure 9. Photographs of the five crop types at the time of the radar acquisition: (a) sugar beet; (b) winter wheat; (c) maize; (d) rape; and (e) winter barley.

In this paper, we will concentrate on the following fields (see location in Figure 8):

(1) Sugar beet: Fields 102 and 460;

(2) Winter wheat: Fields 230 and 250;

(3) Maize: Field 222;

(4) Rape: Fields 101, 110, 130, and 140; and

(5) Winter barley: Fields 440 and 450.

Sugar beet: On the day of radar acquisition, Field 102 is a bare soil with few vegetation residual and Field 460 is a completely bare soil. The decomposition power contributions and best-fit volume scattering models for the two fields are shown in Tables 5 and 6 respectively. It can be seen that, as expected, surface scattering is the dominant scattering mechanism in all the decomposition methods. Compared with $\mathrm{Y} 4 \mathrm{O}$ method, the volume scattering components of the other five methods are 
smaller because of the orientation compensation. The double-bounce scattering components of Chen's and proposed method increase and the volume scattering components decrease, respectively, when compared with other traditional methods. In addition, the proposed method maintains a high surface scattering component, similar to the traditional methods, whereas that of Chen's method is the lowest one. For these two fields, the most used best-fit volume scattering models for Chen's and the proposed method are the vertical dipoles model. However, because of the residuals, the results in the traditional methods show almost all vertical dipoles model in Field 102, whereas the mixture of vertical dipoles model and random dipoles model in Field 460. In general, it can be stated that for all methods there always exists some coupling of either double-bounce or volume components (or both) even in case of a bare rough surface. This suggests some limitations of the Bragg scattering model and its improvements for accounting for depolarization effects even at L-band. This is clearly seen in the volume component for all four traditional methods where it ranges between $17 \%$ and $24 \%$ of the total backscattered power. Otherwise the double-bounce is negligible in those cases. On the other hand, Chen's and the proposed methods tend to balance the "residual" power not assigned to surface scattering between double-bounce and volume scattering. This negative effect is even more noticeable in the original method by Chen.

Winter Wheat: On that date, the winter wheat is in the early vegetative stages with an average height about 17-18 cm. It can be seen from Tables 7 and 8 that a mixture of scattering components appears in the two fields from all decomposition methods. For both wheat fields, the best-fit volume scattering model for the traditional methods is the random dipoles model, the proposed method mostly chooses the vertical dipoles model, whereas Chen's method mostly adopts the horizontal and vertical dipoles model. In addition, it must also be noted that the retrieved strongest scattering mechanism on Field 230 for the traditional methods is the direct surface whereas it happens to be the double-bounce for Chen's and new methods. Otherwise, on Field 250, which is located at far range, the scattering mechanisms tend to be more similar among all them since the increase of the volume component leads to a redistribution of the power among mechanisms. This agrees with the expected behavior related to a higher incidence angle.

Maize: From Figure 9, we can see that the maize field at that date is a bare soil with some vegetation residuals. In Table 9, it is obvious that the dominant contribution in this field is surface scattering for all methods. The mostly used best-fit volume scattering model in traditional methods is the random dipoles model, whereas in Chen's and the proposed methods is the vertical dipoles model. More interestingly, as happens for sugar beet fields (especially Field 102), the double-bounce is negligible (as expected) but, however, the volume scattering is $22.6 \%$ of the total power for Y4R, S4R, and G4U. While being a lower power than that provided by $Y 4 \mathrm{O}$ it becomes evident that none of the traditional methods is able to properly cope with the overestimation of volume component which seems to be an undesirable and essential attribute of these type of decompositions. In case of Chen's and more clearly in the proposed method, it can be interpreted that they are able to diminish this drawback since it seems they tend to redistribute the residual power between double-bounce and volume scattering as it happened in sugar beet field. Notwithstanding this improvement, there is still $25 \%$ of the backscattered power in the best case (which is the proposed method) that is assigned to scattering mechanisms not expected on a bare surface at L-band. This effect clearly points out a recurrent flaw that must be further investigated. In this regard, the different scattering model choices in both traditional and Chen's and proposed methods can be also indicative on the role that volume component could play in the decomposition as they could be acting as mere fitting components.

Rape: Results for four rape fields are shown in Tables 10-13, respectively. As an overall comment beyond the numerical differences among methods, these results epitomize the noticeable disagreement between both types of decomposition methodologies. As shown, in rape Fields 110, 130, and 140, the traditional methods show similar levels of both surface scattering and volume with higher percentages than double-bounce scattering which represents $11 \%$ of total power at most. The least frequently used volume scattering model in these three fields is the vertical dipoles model, whereas the 
most frequently chosen volume model alternates between horizontal dipoles and randomly oriented ones. On the other hand, Chen's and proposed methods retrieve in Fields 110, 130, and 140 a mixture of three scattering mechanisms without an obvious dominant scattering mechanism. According to these results and even though we have some knowledge of the status of rape fields on that date (see Figure 9) we cannot provide any conclusive statement on which decomposition methodology characterizes better the scattering processes on rape Fields 110, 130, and 140. Considering now the rape Field 101 located at the furthest point from nadir, a different decomposition behavior is observed. In all methods, volume scattering is the dominant scattering component, which is consistent with a shallower incidence since the sensor becomes more sensitive to the volume. However, the volume power for the traditional methods is about $72 \%$, which is clearly higher than $56 \%$ for Chen's method or $62 \%$ for the proposed one. Regarding the selection of the type of volume model, there is again an obvious disagreement among the set of traditional methods, Chen's and the proposed method. Among all them, the vertical dipoles model is hardly ever chosen.

Winter barley: From Table 14, it can be seen that in Field 440 the surface scattering and volume scattering are the two dominant scattering mechanisms in traditional methods, whereas Chen's and proposed methods exhibit double-bounce and volume scattering mechanisms as the two strongest mechanisms but the surface component is also noticeable. On the other hand, for Field 450 whose results are shown in Table 15, all methods retrieve surface and double-bounce scattering as the two main scattering components. In this regard, both sets of techniques yield the same qualitative description for this field. In addition, for both fields, the mostly used volume scattering model in traditional methods is the random dipoles model, whereas in Chen's and proposed methods are the horizontal and vertical dipoles model, respectively. It is important to emphasize that the incidence angle has hardly changed between Fields 440 and 450. This allows stating that: (1) all different decomposition methods adapt to the structural changes of soil and plants; and (2) whether these disagreements are due to design flaws on either one group of methods or the other or both cannot be ascertained according to the present study.

Forest: We tested two forest areas in the image, one in the near-range region and the other in the far-range region. From the results in Tables 16 and 17, it is clear that volume scattering is the dominant scattering mechanism in these two forest areas in all methods, as expected. It is noted that in the traditional decomposition processing, the power of the surface and double-bounce scattering components are forced to be zero when the sum of the volume and helix scattering components is over the total span. Then the decomposition algorithm jumps to a two-component decomposition and the volume scattering will be directly calculated by just using the difference of total span and the helix power. For the forest area in near-range, after computation, there are 85.29\%, 39.78\%, $39.78 \%$, and $39.78 \%$ of pixels in Y4O, Y4R, S4R, and G4U methods, respectively, in which this happens. For the forest area in the far-range, the percentages are $81.70 \%, 34.90 \%, 34.90 \%, 34.90 \%$, respectively. Note that as the results of Chen's and proposed methods are the optimized and adapted solutions by the algorithm, they do not suffer from this limitation. In addition, it must be highlighted that the random dipoles model is the mostly used volume scattering model in traditional decomposition methods, whereas the entropy model is the mostly selected volume scattering model in both Chen's and proposed methods. It is also pointed out that some contribution of double-bounce mechanism is also expected from forest areas at L-band according to a number of previous works in the literature. As shown, Chen's and the proposed methods fulfill this expectation better than all four traditional methods. However, at this moment we cannot provide any conclusive statement on this issue.

Building: According to the results, as expected, it is evident from Table 18 that the double-bounce scattering is the dominant component in all methods. The volume scattering components in S4R and G4U are reduced because of the effect of the selection of the volume model caused by the oriented dihedral structures. The mostly used volume model in Chen's and proposed methods is the entropy model, and the volume scattering component is further reduced compared with the traditional method. Finally, the proposed method shows the highest double-bounce scattering contribution, which demonstrates its consistency also for build-up area. 
Table 5. Decomposition mean power statistics and volume scattering models statistics over the sugar beet Field 102.

\begin{tabular}{cccccccccc}
\hline Method & $\begin{array}{c}\text { Ps } \\
\mathbf{( \% )}\end{array}$ & $\begin{array}{c}\text { Pd } \\
\mathbf{( \% )}\end{array}$ & $\begin{array}{c}\text { Pv } \\
\mathbf{( \% )}\end{array}$ & $\begin{array}{c}\mathbf{P c} \\
\mathbf{( \% )}\end{array}$ & $\begin{array}{c}\text { Tv_h } \\
\mathbf{( \% )}\end{array}$ & $\begin{array}{c}\text { Tv_v } \\
\mathbf{( \% )}\end{array}$ & $\begin{array}{c}\text { Tv_r } \\
\mathbf{( \% )}\end{array}$ & $\begin{array}{c}\text { Tv_Dihedral } \\
\text { (\%) }\end{array}$ & $\begin{array}{c}\text { Tv_Entropy } \\
(\mathbf{\%})\end{array}$ \\
\hline Y4O & 78.99 & 1.05 & 18.49 & 1.47 & 0 & 99.71 & 0.29 & - & - \\
Y4R & 79.59 & 1.42 & 17.52 & 1.47 & 0 & 99.71 & 0.29 & - & - \\
S4R & 79.59 & 1.42 & 17.52 & 1.47 & 0 & 99.71 & 0.29 & 0 & - \\
G4U & 80.56 & 0.45 & 17.52 & 1.47 & 0 & 99.71 & 0.29 & 0 & - \\
Chen & 63.97 & 21.13 & 13.84 & 1.06 & 10.4 & 85.71 & 2.58 & - & 1.31 \\
Proposed & 78.81 & 9.48 & 10.24 & 1.46 & 2.88 & 94.80 & 1.19 & - & 1.13 \\
\hline
\end{tabular}

Table 6. Decomposition mean power statistics and volume scattering models statistics over the sugar beet Field 460 .

\begin{tabular}{cccccccccc}
\hline Method & $\begin{array}{c}\text { Ps } \\
\mathbf{( \% )}\end{array}$ & $\begin{array}{c}\mathbf{P d} \\
\mathbf{( \% )}\end{array}$ & $\begin{array}{c}\mathbf{P v} \\
\mathbf{( \% )}\end{array}$ & $\begin{array}{c}\text { Pc } \\
\mathbf{( \% )}\end{array}$ & $\begin{array}{c}\text { Tv_h } \\
\mathbf{( \% )}\end{array}$ & $\begin{array}{c}\text { Tv_v } \\
(\mathbf{\%})\end{array}$ & $\begin{array}{c}\text { Tv_r } \\
(\mathbf{\%})\end{array}$ & $\begin{array}{c}\text { Tv_Dihedral } \\
(\mathbf{\%})\end{array}$ & $\begin{array}{c}\text { Tv_Entropy } \\
(\mathbf{\%})\end{array}$ \\
\hline Y4O & 71.33 & 2.44 & 24.24 & 1.99 & 0.01 & 52.45 & 47.55 & - & - \\
Y4R & 73.37 & 5.84 & 18.80 & 1.99 & 0.04 & 47.68 & 52.28 & - & - \\
S4R & 73.79 & 5.87 & 18.35 & 1.99 & 0.02 & 47.65 & 52.12 & 0.21 & - \\
G4U & 75.01 & 4.65 & 18.35 & 1.99 & 0.02 & 47.65 & 52.12 & 0.21 & - \\
Chen & 61.08 & 21.50 & 15.70 & 1.73 & 25.57 & 72.30 & 0.99 & - & 1.14 \\
Proposed & 70.69 & 14.63 & 12.69 & 1.98 & 25.64 & 66.06 & 6.72 & - & 1.57 \\
\hline
\end{tabular}

Table 7. Decomposition mean power statistics and volume scattering models statistics over the winter wheat Field 230.

\begin{tabular}{cccccccccc}
\hline Method & $\begin{array}{c}\text { Ps } \\
\mathbf{( \% )}\end{array}$ & $\begin{array}{c}\text { Pd } \\
\mathbf{( \% )}\end{array}$ & $\begin{array}{c}\mathbf{P v} \\
\mathbf{( \% )}\end{array}$ & $\begin{array}{c}\text { Pc } \\
\mathbf{( \% )}\end{array}$ & $\begin{array}{c}\text { Tv_h } \\
\mathbf{( \% )}\end{array}$ & $\begin{array}{c}\text { Tv_v } \\
\mathbf{( \% )}\end{array}$ & $\begin{array}{c}\text { Tv_r } \\
\mathbf{( \% )}\end{array}$ & $\begin{array}{c}\text { Tv_Dihedral } \\
(\mathbf{\%})\end{array}$ & $\begin{array}{c}\text { Tv_Entropy } \\
(\mathbf{\%})\end{array}$ \\
\hline Y4O & 37.99 & 28.73 & 29.88 & 3.41 & 4.30 & 2.73 & 92.97 & - & - \\
Y4R & 38.54 & 29.26 & 28.79 & 3.41 & 4.28 & 2.79 & 92.93 & - & - \\
S4R & 40.01 & 29.37 & 27.21 & 3.41 & 2.74 & 2.66 & 82.31 & 12.29 & - \\
G4U & 40.24 & 29.14 & 27.21 & 3.41 & 2.74 & 2.66 & 82.31 & 12.29 & - \\
Chen & 33.19 & 37.77 & 25.69 & 3.36 & 35.78 & 34.93 & 14.68 & - & 14.61 \\
Proposed & 30.83 & 40.58 & 25.18 & 3.41 & 10.25 & 63.16 & 6.03 & - & 20.56 \\
\hline
\end{tabular}

Table 8. Decomposition mean power statistics and volume scattering models statistics over the winter wheat Field 250.

\begin{tabular}{cccccccccc}
\hline Method & $\begin{array}{c}\mathbf{P s} \\
\mathbf{( \% )}\end{array}$ & $\begin{array}{c}\mathbf{P d} \\
\mathbf{( \% )}\end{array}$ & $\begin{array}{c}\mathbf{P v} \\
\mathbf{( \% )}\end{array}$ & $\begin{array}{c}\mathbf{P c} \\
\mathbf{( \% )}\end{array}$ & $\begin{array}{c}\text { Tv_h } \\
\mathbf{( \% )}\end{array}$ & $\begin{array}{c}\text { Tv_v } \\
\mathbf{( \% )}\end{array}$ & $\begin{array}{c}\text { Tv_r } \\
\mathbf{( \% )}\end{array}$ & $\begin{array}{c}\text { Tv_Dihedral } \\
(\mathbf{\%})\end{array}$ & $\begin{array}{c}\text { Tv_Entropy } \\
(\mathbf{\%})\end{array}$ \\
\hline Y4O & 21.14 & 31.11 & 42.36 & 5.39 & 0.22 & 38.62 & 61.16 & - & - \\
Y4R & 22.55 & 32.91 & 39.15 & 5.39 & 0.33 & 38.00 & 61.67 & - & - \\
S4R & 27.62 & 33.69 & 33.30 & 5.39 & 0.15 & 25.11 & 39.01 & 35.74 & - \\
G4U & 27.38 & 33.93 & 33.30 & 5.39 & 0.15 & 25.11 & 39.01 & 35.74 & - \\
Chen & 29.07 & 35.32 & 30.38 & 5.23 & 43.74 & 23.93 & 7.30 & - & 25.03 \\
Proposed & 27.96 & 35.67 & 30.99 & 5.38 & 5.01 & 73.36 & 1.20 & - & 20.43 \\
\hline
\end{tabular}

Table 9. Decomposition mean power statistics and volume scattering models statistics over the maize Field 222.

\begin{tabular}{cccccccccc}
\hline Method & $\begin{array}{c}\text { Ps } \\
\mathbf{( \% )}\end{array}$ & $\begin{array}{c}\text { Pd } \\
\mathbf{( \% )}\end{array}$ & $\begin{array}{c}\mathbf{P v} \\
\mathbf{( \% )}\end{array}$ & $\begin{array}{c}\text { Pc } \\
\mathbf{( \% )}\end{array}$ & $\begin{array}{c}\text { Tv_h } \\
\mathbf{( \% )}\end{array}$ & $\begin{array}{c}\text { Tv_v } \\
(\mathbf{\%})\end{array}$ & $\begin{array}{c}\text { Tv_r } \\
\mathbf{( \% )}\end{array}$ & $\begin{array}{c}\text { Tv_Dihedral } \\
(\mathbf{\%})\end{array}$ & $\begin{array}{c}\text { Tv_Entropy } \\
(\mathbf{\%})\end{array}$ \\
\hline Y4O & 68.98 & 0.17 & 29.53 & 1.33 & 0 & 46.49 & 53.51 & - & - \\
Y4R & 74.18 & 1.89 & 22.6 & 1.33 & 0 & 35.06 & 64.94 & - & - \\
S4R & 74.18 & 1.89 & 22.6 & 1.33 & 0 & 35.06 & 64.94 & 0 & - \\
G4U & 74.41 & 1.66 & 22.6 & 1.33 & 0 & 35.06 & 64.94 & 0 & - \\
Chen & 64.72 & 18.49 & 15.94 & 0.86 & 29.84 & 65.76 & 2.52 & - & 1.87 \\
Proposed & 72.41 & 11.45 & 14.83 & 1.32 & 8.40 & 84.68 & 2.38 & - & 4.55 \\
\hline
\end{tabular}


Table 10. Decomposition mean power statistics and volume scattering models statistics over the rape Field 101.

\begin{tabular}{cccccccccc}
\hline Method & $\begin{array}{c}\text { Ps } \\
\mathbf{( \% )}\end{array}$ & $\begin{array}{c}\mathbf{P d} \\
\mathbf{( \% )}\end{array}$ & $\begin{array}{c}\mathbf{P v} \\
\mathbf{( \% )}\end{array}$ & $\begin{array}{c}\text { Pc } \\
\mathbf{( \% )}\end{array}$ & $\begin{array}{c}\text { Tv_h } \\
\mathbf{( \% )}\end{array}$ & $\begin{array}{c}\text { Tv_v } \\
\mathbf{( \% )}\end{array}$ & $\begin{array}{c}\text { Tv_r } \\
(\mathbf{\%})\end{array}$ & $\begin{array}{c}\text { Tv_Dihedral } \\
(\mathbf{\%})\end{array}$ & $\begin{array}{c}\text { Tv_Entropy } \\
(\mathbf{\%})\end{array}$ \\
\hline Y4O & 12.38 & 5.79 & 77.37 & 4.46 & 42.82 & 0.05 & 57.13 & - & - \\
Y4R & 14.74 & 8.16 & 72.64 & 4.46 & 35.52 & 0.08 & 64.40 & - & - \\
S4R & 14.74 & 8.16 & 72.64 & 4.46 & 35.52 & 0.08 & 64.40 & 0 & - \\
G4U & 15.17 & 7.73 & 72.64 & 4.46 & 35.52 & 0.08 & 64.40 & 0 & - \\
Chen & 16.45 & 22.64 & 56.67 & 4.25 & 35.65 & 27.81 & 12.54 & - & 24.00 \\
Proposed & 12.86 & 20.58 & 62.08 & 4.47 & 42.98 & 3.74 & 28.91 & - & 24.37 \\
\hline
\end{tabular}

Table 11. Decomposition mean power statistics and volume scattering models statistics over the rape Field 110.

\begin{tabular}{cccccccccc}
\hline Method & $\begin{array}{c}\text { Ps } \\
\mathbf{( \% )}\end{array}$ & $\begin{array}{c}\text { Pd } \\
\mathbf{( \% )}\end{array}$ & $\begin{array}{c}\mathbf{P v} \\
\mathbf{( \% )}\end{array}$ & $\begin{array}{c}\text { Pc } \\
\mathbf{( \% )}\end{array}$ & $\begin{array}{c}\text { Tv_h } \\
\mathbf{( \% )}\end{array}$ & $\begin{array}{c}\text { Tv_v } \\
\mathbf{( \% )}\end{array}$ & $\begin{array}{c}\text { Tv_r } \\
(\mathbf{\%})\end{array}$ & $\begin{array}{c}\text { Tv_Dihedral } \\
(\mathbf{\%})\end{array}$ & $\begin{array}{c}\text { Tv_Entropy } \\
(\%)\end{array}$ \\
\hline Y4O & 45.73 & 10.60 & 40.64 & 3.03 & 59.02 & 1.95 & 39.03 & - & - \\
Y4R & 46.41 & 11.18 & 39.38 & 3.03 & 57.44 & 2.06 & 40.50 & - & - \\
S4R & 46.41 & 11.18 & 39.38 & 3.03 & 57.44 & 2.06 & 40.50 & 0 & - \\
G4U & 46.81 & 10.78 & 39.38 & 3.03 & 57.44 & 2.06 & 40.50 & 0 & - \\
Chen & 35.96 & 26.71 & 34.47 & 2.87 & 34.26 & 42.68 & 11.25 & - & 11.81 \\
Proposed & 32.73 & 28.20 & 36.04 & 3.03 & 65.91 & 14.32 & 12.56 & - & 7.22 \\
\hline
\end{tabular}

Table 12. Decomposition mean power statistics and volume scattering models statistics over the rape Field 130.

\begin{tabular}{cccccccccc}
\hline Method & $\begin{array}{c}\text { Ps } \\
\mathbf{( \% )}\end{array}$ & $\begin{array}{c}\text { Pd } \\
\mathbf{( \% )}\end{array}$ & $\begin{array}{c}\text { Pv } \\
\mathbf{( \% )}\end{array}$ & $\begin{array}{c}\text { Pc } \\
\mathbf{( \% )}\end{array}$ & $\begin{array}{c}\text { Tv_h } \\
\mathbf{( \% )}\end{array}$ & $\begin{array}{c}\text { Tv_v } \\
(\mathbf{\%})\end{array}$ & $\begin{array}{c}\text { Tv_r } \\
\mathbf{( \% )}\end{array}$ & $\begin{array}{c}\text { Tv_Dihedral } \\
(\mathbf{\%})\end{array}$ & $\begin{array}{c}\text { Tv_Entropy } \\
(\mathbf{\%})\end{array}$ \\
\hline Y4O & 45.65 & 3.86 & 47.07 & 3.42 & 18.63 & 5.25 & 76.12 & - & - \\
Y4R & 48.51 & 5.01 & 43.05 & 3.42 & 17.12 & 6.46 & 76.42 & - & - \\
S4R & 48.51 & 5.01 & 43.05 & 3.42 & 17.12 & 6.46 & 76.42 & 0 & - \\
G4U & 48.46 & 5.06 & 43.05 & 3.42 & 17.12 & 6.46 & 76.42 & 0 & - \\
Chen & 26.58 & 37.8 & 32.78 & 2.85 & 31.77 & 60.04 & 4.25 & - & 3.94 \\
Proposed & 33.20 & 31.57 & 31.83 & 3.40 & 25.77 & 50.62 & 10.41 & - & 13.20 \\
\hline
\end{tabular}

Table 13. Decomposition mean power statistics and volume scattering models statistics over the rape Field 140.

\begin{tabular}{|c|c|c|c|c|c|c|c|c|c|}
\hline Method & $\begin{array}{l}\text { Ps } \\
(\%)\end{array}$ & $\begin{array}{l}P d \\
(\%)\end{array}$ & $\begin{array}{l}\mathrm{Pv} \\
(\%)\end{array}$ & $\begin{array}{l}\text { Pc } \\
(\%)\end{array}$ & $\begin{array}{c}\text { Tv_h } \\
(\%)\end{array}$ & $\begin{array}{c}\text { Tv_v } \\
(\%)\end{array}$ & $\begin{array}{c}\text { Tv_r } \\
(\%)\end{array}$ & $\begin{array}{c}\text { Tv_Dihedral } \\
(\%)\end{array}$ & $\begin{array}{c}\text { Tv_Entropy } \\
(\%)\end{array}$ \\
\hline $\mathrm{Y} 4 \mathrm{O}$ & 49.43 & 2.21 & 45.36 & 3.00 & 65.36 & 0 & 34.64 & - & - \\
\hline S4R & 50.50 & 2.85 & 43.65 & 3.00 & 62.15 & 0 & 37.85 & 0 & - \\
\hline G4U & 50.40 & 2.95 & 43.65 & 3.00 & 62.15 & 0 & 37.85 & 0 & - \\
\hline Chen & 30.76 & 33.64 & 33.31 & 2.28 & 48.70 & 49.11 & 1.68 & - & 0.50 \\
\hline
\end{tabular}

Table 14. Decomposition mean power statistics and volume scattering models statistics over the winter barley Field 440 .

\begin{tabular}{cccccccccc}
\hline Method & $\begin{array}{c}\mathbf{P s} \\
\mathbf{( \% )}\end{array}$ & $\begin{array}{c}\mathbf{P d} \\
\mathbf{( \% )}\end{array}$ & $\begin{array}{c}\mathbf{P v} \\
\mathbf{( \% )}\end{array}$ & $\begin{array}{c}\mathbf{P c} \\
\mathbf{( \% )}\end{array}$ & $\begin{array}{c}\text { Tv_h } \\
\mathbf{( \% )}\end{array}$ & $\begin{array}{c}\text { Tv_v } \\
\mathbf{( \% )}\end{array}$ & $\begin{array}{c}\text { Tv_r } \\
\mathbf{( \% )}\end{array}$ & $\begin{array}{c}\text { Tv_Dihedral } \\
(\mathbf{\%})\end{array}$ & $\begin{array}{c}\text { Tv_Entropy } \\
(\mathbf{\%})\end{array}$ \\
\hline Y4O & 46.14 & 8.75 & 42.00 & 3.11 & 0.43 & 4.51 & 95.06 & - & - \\
Y4R & 46.72 & 9.32 & 40.85 & 3.11 & 0.43 & 4.66 & 94.90 & - & - \\
S4R & 46.72 & 9.32 & 40.85 & 3.11 & 0.43 & 4.66 & 94.90 & 0 & - \\
G4U & 47.31 & 8.73 & 40.85 & 3.11 & 0.43 & 4.66 & 94.90 & 0 & - \\
Chen & 25.13 & 37.09 & 34.90 & 2.88 & 49.52 & 36.57 & 5.77 & - & 8.14 \\
Proposed & 27.00 & 35.56 & 34.35 & 3.10 & 1.22 & 91.76 & 2.28 & - & 4.74 \\
\hline
\end{tabular}


Table 15. Decomposition mean power statistics and volume scattering models statistics over the winter barley Field 450 .

\begin{tabular}{cccccccccc}
\hline Method & $\begin{array}{c}\text { Ps } \\
\mathbf{( \% )}\end{array}$ & $\begin{array}{c}\text { Pd } \\
\mathbf{( \% )}\end{array}$ & $\begin{array}{c}\text { Pv } \\
\mathbf{( \% )}\end{array}$ & $\begin{array}{c}\text { Pc } \\
\mathbf{( \% )}\end{array}$ & $\begin{array}{c}\text { Tv_h } \\
\mathbf{( \% )}\end{array}$ & $\begin{array}{c}\text { Tv_v } \\
\mathbf{( \% )}\end{array}$ & $\begin{array}{c}\text { Tv_r } \\
\mathbf{( \% )}\end{array}$ & $\begin{array}{c}\text { Tv_Dihedral } \\
\mathbf{( \% )}\end{array}$ & $\begin{array}{c}\text { Tv_Entropy } \\
(\mathbf{\%})\end{array}$ \\
\hline Y4O & 51.24 & 24.40 & 17.74 & 6.62 & 0.04 & 27.22 & 72.74 & - & - \\
Y4R & 51.83 & 25.12 & 16.49 & 6.56 & 0.04 & 28.64 & 71.32 & - & - \\
S4R & 51.94 & 25.13 & 16.37 & 6.56 & 0.04 & 28.36 & 69.71 & 1.89 & - \\
G4U & 51.88 & 25.19 & 16.37 & 6.56 & 0.04 & 28.36 & 69.71 & 1.89 & - \\
Chen & 37.02 & 42.32 & 14.02 & 6.65 & 30.84 & 29.01 & 16.44 & - & 23.71 \\
Proposed & 39.93 & 39.83 & 17.72 & 6.52 & 2.54 & 88.17 & 1.12 & - & 8.17 \\
\hline
\end{tabular}

Table 16. Decomposition mean power statistics and volume scattering models statistics over the near-range forest area.

\begin{tabular}{cccccccccc}
\hline Method & $\begin{array}{c}\text { Ps } \\
\mathbf{( \% )}\end{array}$ & $\begin{array}{c}\text { Pd } \\
\mathbf{( \% )}\end{array}$ & $\begin{array}{c}\text { Pv } \\
\mathbf{( \% )}\end{array}$ & $\begin{array}{c}\text { Pc } \\
\mathbf{( \% )}\end{array}$ & $\begin{array}{c}\text { Tv_h } \\
(\mathbf{\%})\end{array}$ & $\begin{array}{c}\text { Tv_v } \\
\mathbf{( \% )}\end{array}$ & $\begin{array}{c}\text { Tv_r } \\
(\mathbf{\%})\end{array}$ & $\begin{array}{c}\text { Tv_Dihedral } \\
(\mathbf{\%})\end{array}$ & $\begin{array}{c}\text { Tv_Entropy } \\
(\mathbf{\%})\end{array}$ \\
\hline Y4O & 0.22 & 1.35 & 93.49 & 4.94 & 34.63 & 0.03 & 65.34 & - & - \\
Y4R & 1.25 & 5.77 & 88.05 & 4.94 & 21.07 & 0.54 & 78.39 & - & - \\
S4R & 1.31 & 5.82 & 87.93 & 4.94 & 20.79 & 0.54 & 78.38 & 0.30 & - \\
G4U & 1.26 & 5.87 & 87.93 & 4.94 & 20.79 & 0.54 & 78.38 & 0.30 & - \\
Chen & 14.24 & 16.17 & 64.73 & 4.86 & 11.17 & 8.15 & 2.62 & - & 78.06 \\
Proposed & 2.98 & 14.46 & 77.57 & 4.99 & 17.95 & 0.71 & 35.04 & & 46.30 \\
\hline
\end{tabular}

Table 17. Decomposition mean power statistics and volume scattering models statistics over the far-range forest area.

\begin{tabular}{cccccccccc}
\hline Method & $\begin{array}{c}\text { Ps } \\
\mathbf{( \% )}\end{array}$ & $\begin{array}{c}\mathbf{P d} \\
\mathbf{( \% )}\end{array}$ & $\begin{array}{c}\mathbf{P v} \\
\mathbf{( \% )}\end{array}$ & $\begin{array}{c}\mathbf{P c} \\
\mathbf{( \% )}\end{array}$ & $\begin{array}{c}\text { Tv_h } \\
\mathbf{( \% )}\end{array}$ & $\begin{array}{c}\text { Tv_v } \\
\mathbf{( \% )}\end{array}$ & $\begin{array}{c}\text { Tv_r } \\
(\mathbf{\%})\end{array}$ & $\begin{array}{c}\text { Tv_Dihedral } \\
(\mathbf{\%})\end{array}$ & $\begin{array}{c}\text { Tv_Entropy } \\
(\mathbf{\%})\end{array}$ \\
\hline Y4O & 0.24 & 1.98 & 91.95 & 5.83 & 46.45 & 2.39 & 51.16 & - & - \\
Y4R & 1.11 & 6.84 & 86.22 & 5.83 & 28.86 & 2.41 & 68.73 & - & - \\
S4R & 1.14 & 6.84 & 86.19 & 5.83 & 28.86 & 2.37 & 68.65 & 0.13 & - \\
G4U & 1.04 & 6.94 & 86.19 & 5.83 & 28.86 & 2.37 & 68.65 & 0.13 & - \\
Chen & 14.83 & 18.3 & 61.10 & 5.77 & 14.30 & 8.58 & 2.54 & - & 74.58 \\
Proposed & 3.15 & 16.02 & 74.94 & 5.89 & 32.14 & 3.71 & 23.06 & - & 41.10 \\
\hline
\end{tabular}

Table 18. Decomposition mean power statistics and volume scattering models statistics over the building area.

\begin{tabular}{cccccccccc}
\hline Method & $\begin{array}{c}\text { Ps } \\
\mathbf{( \% )}\end{array}$ & $\begin{array}{c}\mathbf{P d} \\
\mathbf{( \% )}\end{array}$ & $\begin{array}{c}\mathbf{P v} \\
\mathbf{( \% )}\end{array}$ & $\begin{array}{c}\mathbf{P c} \\
\mathbf{( \% )}\end{array}$ & $\begin{array}{c}\text { Tv_h } \\
\mathbf{( \% )}\end{array}$ & $\begin{array}{c}\text { Tv_v } \\
\mathbf{( \% )}\end{array}$ & $\begin{array}{c}\text { Tv_r } \\
\mathbf{( \% )}\end{array}$ & $\begin{array}{c}\text { Tv_Dihedral } \\
\mathbf{( \% )}\end{array}$ & $\begin{array}{c}\text { Tv_Entropy } \\
(\mathbf{\%})\end{array}$ \\
\hline Y4O & 11.26 & 62.31 & 21.99 & 4.44 & 67.78 & 5.01 & 27.21 & - & - \\
Y4R & 11.85 & 65.05 & 19.24 & 3.86 & 67.28 & 6.00 & 26.72 & - & - \\
S4R & 14.22 & 65.93 & 15.98 & 3.86 & 38.56 & 5.28 & 21.92 & 34.24 & - \\
G4U & 14.08 & 66.08 & 15.98 & 3.86 & 38.56 & 5.28 & 21.92 & 34.24 & - \\
Chen & 24.54 & 57.91 & 11.37 & 6.17 & 27.23 & 28.93 & 8.14 & - & 35.70 \\
Proposed & 8.97 & 71.60 & 15.58 & 3.86 & 27.94 & 23.92 & 14.69 & - & 33.45 \\
\hline
\end{tabular}




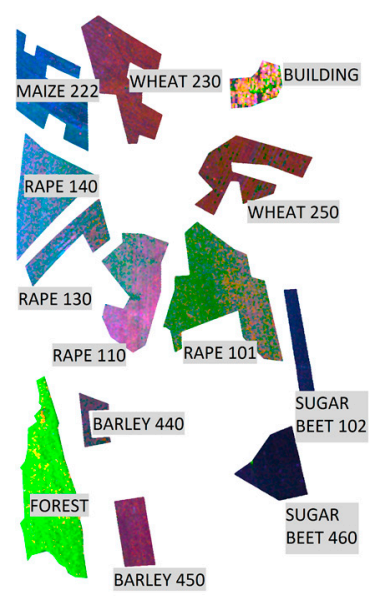

(a)

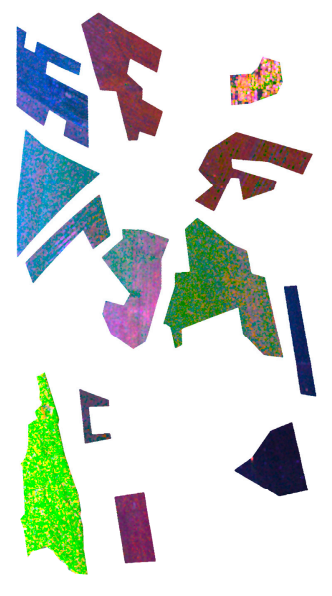

(b)

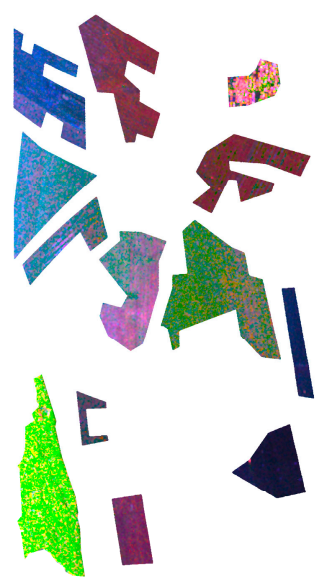

(c)

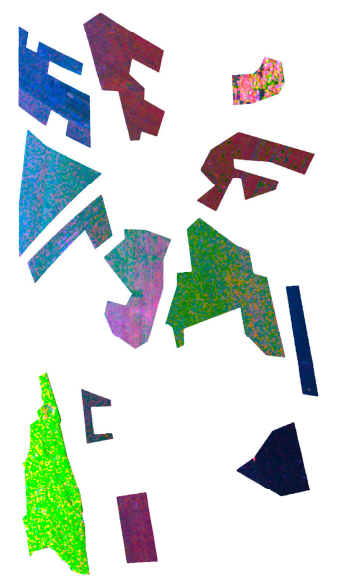

(d)

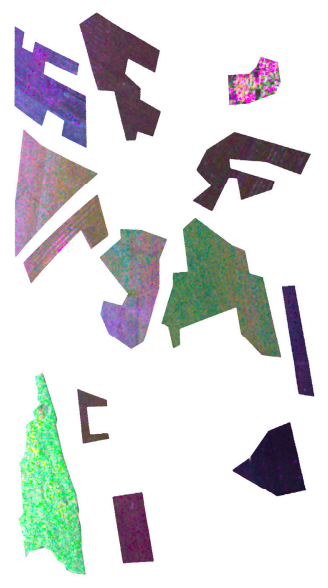

(e)

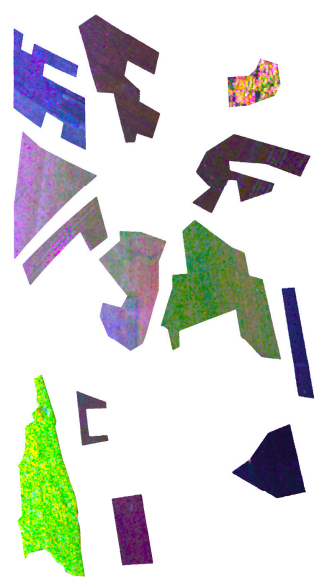

(f)

Figure 10. Decomposition results from different methods: (a) Y4O decomposition; (b) Y4R decomposition; (c) S4R decomposition; (d) G4U decomposition; (e) Chen decomposition; and (f) Proposed decomposition. The images are colored by Pd (red), Pv (green), and Ps (blue).

If we analyze the whole scene within the defined regions of interest (i.e., crops, forests and built-up areas), the mean values of the normalized minimum residuals $R_{\min }$ are 0.0020 and 0.0062 for Chen's 
and the proposed method, respectively. The values are both low enough, even though the proposed method shows a larger residual because of the tighter boundary conditions.

Regarding the retrieved values of $\beta$, the feasible range according to the model (Figure 3 ) is from -0.5695 to -0.0516 for our tested E-SAR data. Figure 11 shows the histograms of the values of $\beta$ in all fields from all different methods. Since the traditional methods impose no limitation on the final retrievals and we found the values of some pixels are very high or low, in order to show the histograms, we limit them to the interval $[-1,1]$. It is evident that due to the assumption made in traditional decomposition methods the values of $\beta$ are zero in many cases. On the other side, the result from Chen's method is very likely to fall outside the physical range, whereas the proposed method yields solutions that are distributed in the physical range.

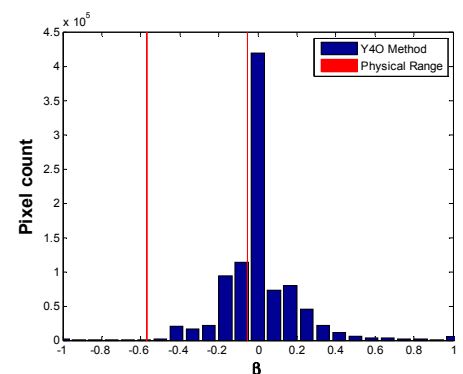

(a)

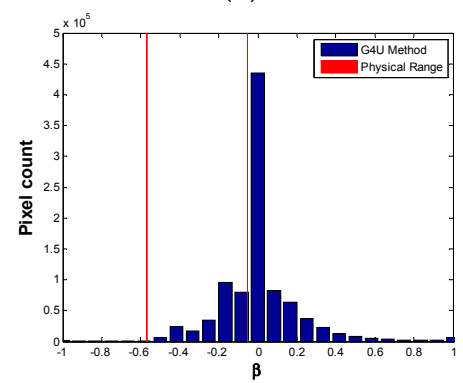

(d)

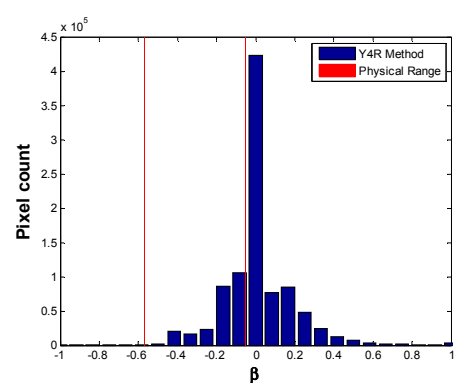

(b)

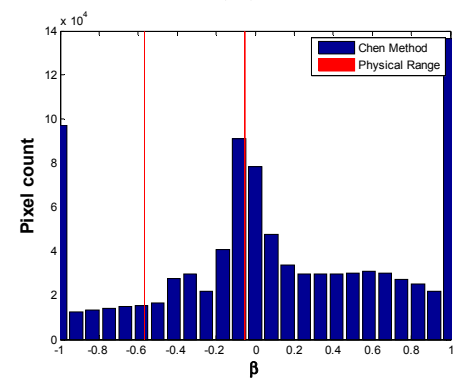

(e)

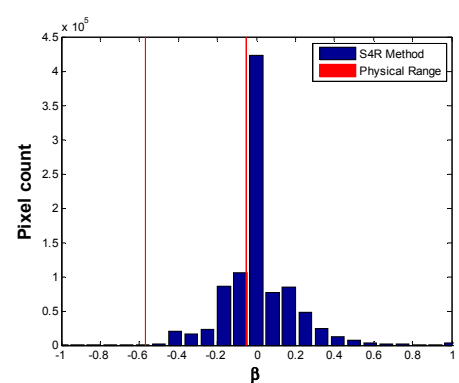

(c)

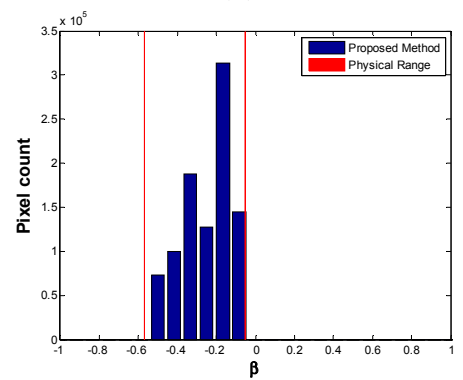

(f)

Figure 11. Histograms of the values of $\beta$ from different methods: (a) Y4O decomposition; (b) Y4R decomposition; (c) S4R decomposition; (d) G4U decomposition; (e) Chen decomposition; and (f) Proposed decomposition. The red lines denote the feasible range according to the model.

For $\alpha$, because of adopting the magnitude and angle of $\alpha$ as the unknown parameters in the equations solving system and adding the constraint of the magnitude in the proposed method, there are no cases where the absolute value of $\alpha$ is higher than one. However, $61.01 \%$ of pixels in Chen's method exhibit retrieved $\alpha$ higher than one. This is a consequence of using real and imagery parts of $\alpha$ as unknowns in the nonlinear optimization processing and without adding the constraint of magnitude in the system.

\section{Discussion}

\subsection{Necessity of Checking PolSAR Decomposition Results from Quantitative Perspective}

According to the actual state of the art of PolSAR decomposition techniques, multiple approaches rooted in the Freeman-Durden concept exist, which are approximately consistent among them as long as we are concerned on the type of dominant scattering components. Nevertheless, the estimated values of the physical entities making up the derived models lead to different interpretations, even though the underlying physical principles of all these decomposition approaches are the same. For qualitative applications, e.g., target detection and land classification, the existing decomposition methods are fairly accepted as well established techniques since they have demonstrated their usefulness in radar 
remote sensing applications. In this context, the evaluation of results usually is based on a visual interpretation and identification according to the dominant scattering mechanisms and no further information regarding the rest of the outputs of the decomposition is retrieved. To our knowledge there exist very few cases [31,32] where this type of techniques (i.e., Freeman-Durden concept) was exploited for a quantitative application. Nevertheless, they are primarily focused on soil moisture inversion but they lack the in-depth analysis we propose in this paper.

According to the results of this work (i.e., from simulations and real test) the proposed improvements lead to very similar results obtained by using classical methods from a qualitative perspective. Contrarily, we have shown that these modifications allow an overall improvement of the final estimates of the whole set of parameters. Consequently, as an important conclusion from the present paper, we would like to stress the necessity of checking the quantitative accuracy of model-based PolSAR techniques for a reliable physical description of land covers beyond their proven utility for qualitative features extraction. In addition, two limitations of the present approach must be noted. First, a Gaussian distribution is assumed for the data, which may not be the case for some forest areas exhibiting a K-distribution [3]. Secondly, we make use of a particular kind of matrix structure, which only matches specific kinds of land cover. Hence, these issues prevent the generalization of these conclusions in a wide sense.

\subsection{Selection of the Model-Based Decomposition Method}

The general decomposition framework we chose is the method proposed by Chen et al. [22], where all scattering components contributing to the model in this decomposition method are assigned the same priority in the inversion strategy. The unknowns are simultaneously determined by using a nonlinear least squares optimization without prior subtracting or fixing any of them. Then, we have focused this work on the improvement of the performance of the optimization algorithm in order to obtain physically feasible parameter values.

It is noteworthy to emphasize that Chen's method exploits a number of advances and key ideas that were previously proposed separately in the literature regarding model-based decompositions. Therefore, in our opinion the joint application of those concepts in a proper way makes Chen's approach one of the most complete and general decompositions within the topic since it is capable of dealing with a wider variety of scattering scenarios than any other previous model-based decomposition scheme. Consequently, we chose Chen's method to quantitatively check the accuracy of parameters retrieved from model-based decomposition approaches. It must be noted that the methodology employed in this paper can be applied in a straightforward way to test separately any of the previous existing decomposition methods.

\subsection{Future Research Directions}

Future work should be mainly focused on three aspects. First, it is noted that instead of just nine unknowns, there is actually also an extra variable that depends on the type of volume. In this study, the decomposition framework followed the discretization proposed by Chen et al. by considering four typical cases to select the volume model matrix with minimum residual term in an optimal way. However, in the future more representative volume scattering models together with iterative inversion schemes should be included and/or developed to improve the decomposition performance. In addition, as one reviewer proposes, the inversion approach could be modified by directly replacing, as independent parameters to retrieve, $|\alpha|, \operatorname{Arg}(\alpha)$ and $\beta$ with the dielectric constants and the differential phase. This would involve even more complicated equations but it would directly yield the physical parameters. Second, the ideas discussed in this paper can be exported to the PolInSAR decomposition framework. Third, we followed the classical Monte-Carlo simulation method to simulate PolSAR dataset for quantitative analysis in this study but, however, it is based on the assumption that Chen's decomposition model is general enough for generating the coherency matrix. Certainly, the use of a particular model to simulate the corresponding 
covariance/coherency matrix elements is by definition an intrinsic constraint which hinders the generalization of results. Alternatively, the use of a rigorous $3 \mathrm{D}$ electromagnetic model to compute the second order scattering elements could lead to stronger evidences on the performance of model-based decomposition techniques. Actually, this strategy corresponds to the highest levels of evidence and this should be the future line to follow for a conclusive assessment of target decomposition schemes. However, it is also noted that rigorous electromagnetic modeling also relies on assumptions which can be also subject to multiple criticisms, corrections and adjustments regarding the models involved for quantifying the scattering processes. Hence, this could be an arduous and overwhelming task and, perhaps, this is the reason why all model-based decomposition methods have been mostly applied for qualitative studies so far. Consequently, we think there is gap in model-based decompositions topic that must be filled, which concerns the assessment of all the existing methods. In our opinion, the present paper does not fill completely this gap but it does contribute to do so. Finally, the performance of these methods for a quantitative description of land covers should be further investigated in real scenarios. In this regard, validation tests should be designed to check the accuracy of those parameters useful for practical applications, i.e., effective dielectric constants of depolarizing targets and volume descriptors.

\section{Conclusions}

In this paper, we have addressed the feasibility of parameters retrieved from general polarimetric model-based decompositions from the perspective of quantitative applications. To do so, we have chosen the method recently proposed by Chen et al. since it is one of the most complete and general decompositions published so far. However, this approach exhibits a number of flaws concerning the numerical inversion which prevent in many cases a correct parameter retrieval. In order to obtain more physically feasible decomposition results, we have proposed a new inversion algorithm by revising the way to generate the initial values, by redefining variables boundaries, and by implementing a transformation of variables. Different Monte Carlo simulation tests have shown that the estimation accuracy of all parameters is significantly increased after implementing these changes, although the residuals are slightly increased as a consequence of adding stricter and realistic boundary conditions. Among all the modifications, the redefinition of variables boundaries in agreement with the direct physical model provides the largest contribution for improving the decomposition results.

Fully polarimetric L-band SAR data acquired by E-SAR platform together with ground measurements over an agricultural and forested area from AgriSAR2006 campaign have also been employed to test the performance of the proposed algorithm. Regarding the use of L-band data, it is noted that this a frequency band well suited for a wide variety of vegetated scenarios either with short or tall and dense vegetation. However, attending to the different scattering physics expected for different frequencies, our conclusions regarding the real data analysis must be limited to the employed frequency band. Other higher frequencies such as C- and X-band will certainly yield different responses and should be object of further investigations.

Besides Chen's and the proposed method, other classical incoherent model-based decomposition methods (i.e., Y4O, Y4R, S4R, and G4U) have been also included for the comparative analysis. On the one hand, our analysis on the outputs of both groups of techniques reveals different qualitative descriptions of the same cover, which suggests that additional studies are required to progress in polarimetric decomposition models. On the other hand, some results, such as those provided by the Bragg coefficient ratio (i.e., $\beta$ ), show that, despite the well-known utility of the classical methods for qualitative description and classification of land covers, they are prone to provide wrong numerical inversion results which could prevent any subsequent quantitative characterization of specific areas in the scene. According to the current state of the art, a possible strategy to exploit the existing approaches would be a two-level approach consisting on using the well-known decomposition techniques for qualitative studies (land feature classification) and more specialized approaches designed according to the specificity of the land cover to be monitored for quantitative assessments. 
Acknowledgments: This work was supported by National Natural Science Foundation of China (No. 41531068, 41371335, and 41274010), the Spanish Ministry of Economy and Competitiveness and EU FEDER under Projects TEC2011-28201-C02-02 and TIN2014-55413-C2-2-P, and China Scholarship Council (No.201406370079). The authors would like to thanks the European Space Agency and the German Aerospace Center (DLR) for providing the AgriSAR2006 campaign data. We would like to note that the Matlab implementation of the proposed method is available to download via the URL: http:/ / www.sst.dfists.ua.es. Alternatively, it can also be obtained upon request to the authors.

Author Contributions: Qinghua Xie implemented and developed the proposed methodology, and wrote and revised the paper; J. David Ballester-Berman contributed to design the main idea, supervised the work and revised the paper; Juan M. Lopez-Sanchez supervised the work, contributed some ideas and revised the paper; and Changcheng Wang and Jianjun Zhu contributed to the discussion of the results.

Conflicts of Interest: The authors declare no conflict of interest.

\section{References}

1. Cloude, S.R.; Pottier, E. A review of target decomposition theorems in radar polarimetry. IEEE Trans. Geosci. Remote Sens. 1996, 34, 498-518. [CrossRef]

2. Chen, S.W.; Li, Y.Z.; Wang, X.S.; Xiao, S.P.; Sato, M. Modeling and interpretation of scattering mechanisms in polarimetric synthetic aperture radar: Advances and perspectives. IEEE Signal Process. Mag. 2014, 31, 79-89. [CrossRef]

3. Lee, J.S.; Pottier, E. Polarimetric Radar Imaging: From Basics to Applications; CRC Press: Boca Raton, FL, USA, 2009.

4. Freeman, A.; Durden, S.L. A three-component scattering model for polarimetric SAR data. IEEE Trans. Geosci. Remote Sens. 1998, 36, 963-973. [CrossRef]

5. Yamaguchi, Y.; Moriyama, T.; Ishido, M.; Yamada, H. Four-component scattering model for polarimetric SAR image decomposition. IEEE Trans. Geosci. Remote Sens. 2005, 43, 1699-1706. [CrossRef]

6. Yamaguchi, Y.; Yajima, Y.; Yamada, H. A four-component decomposition of PolSAR images based on the coherency matrix. IEEE Geosci. Remote Sens. Lett. 2006, 3, 292-296. [CrossRef]

7. Yajima, Y.; Yamaguchi, Y.; Sato, R.; Yamada, H.; Boerner, W.-M. POLSAR image analysis of wetlands using a modified four-component scattering power decomposition. IEEE Trans. Geosci. Remote Sens. 2008, 46, 1667-1673. [CrossRef]

8. Ballester-Berman, J.D.; Lopez-Sanchez, J.M. Applying the Freeman-Durden decomposition concept to polarimetric SAR interferometry. IEEE Trans. Geosci. Remote Sens. 2010, 48, 466-479. [CrossRef]

9. An, W.T.; Cui, Y.; Yang, J. Three-component model-based decomposition for polarimetric SAR data. IEEE Trans. Geosci. Remote Sens. 2010, 48, 2732-2739.

10. An, W.; Xie, C.; Yuan, X.; Cui, Y.; Yang, J. Four-component decomposition of polarimetric SAR images with deorientation. IEEE Geosci. Remote Sens. Lett. 2011, 8, 1090-1094. [CrossRef]

11. Arii, M.; Van Zyl, J.J.; Kim, Y. Adaptive model-based decomposition of polarimetric SAR covariance matrices. IEEE Trans. Geosci. Remote Sens. 2011, 49, 1104-1113. [CrossRef]

12. Antropov, O.; Rauste, Y.; Hame, T. Volume scattering modeling in PolSAR decompositions: Study of ALOS PALSAR data over boreal forest. IEEE Trans. Geosci. Remote Sens. 2011, 49, 3838-3848. [CrossRef]

13. Lee, J.S.; Schuler, D.L.; Ainsworth, T.L.; Krogager, E.; Kasilingam, D.; Boerner, W.M. On the estimation of radar polarization orientation shifts induced by terrain slopes. IEEE Trans. Geosci. Remote Sens. 2002, 40, $30-41$.

14. Lee, J.S.; Ainsworth, T.L. The effect of orientation angle compensation on coherency matrix and polarimetric target decompositions. IEEE Trans. Geosci. Remote Sens. 2011, 49, 53-64. [CrossRef]

15. Yamaguchi, Y.; Sato, A.; Boerner, W.M.; Sato, R.; Yamada, H. Four-component scattering power decomposition with rotation of coherency matrix. IEEE Trans. Geosci. Remote Sens. 2011, 49, 2251-2258. [CrossRef]

16. Van Zyl, J.J.; Arii, M.; Kim, Y. Model-based decomposition of polarimetric SAR covariance matrices constrained for nonnegative eigenvalues. IEEE Trans. Geosci. Remote Sens. 2011, 49, 3452-3459. [CrossRef]

17. Cui, Y.; Yamaguchi, Y.; Yang, J.; Park, S.E.; Kobayashi, H.; Singh, G. Three-component power decomposition for polarimetric SAR data based on adaptive volume scatter modeling. Remote Sens. 2012, 4, 1559-1572. [CrossRef] 
18. Sato, A.; Yamaguchi, Y.; Singh, G.; Park, S.E. Four-component scattering power decomposition with extended volume scattering model. IEEE Geosci. Remote Sens. Lett. 2012, 9, 166-170. [CrossRef]

19. Shan, Z.L.; Zhang, H.; Wang, C.; An, W.T.; Wu, T.; Chen, X. Four-component model-based decomposition of polarimetric SAR data for special ground objects. IEEE Geosci. Remote Sens. Lett. 2012, 9, 989-993. [CrossRef]

20. Chen, S.W.; Ohki, M.; Shimada, M.; Sato, M. Deorientation effect investigation for model-based decomposition over oriented built-up areas. IEEE Geosci. Remote Sens. Lett. 2013, 10, 273-277. [CrossRef]

21. Singh, G.; Yamaguchi, Y.; Park, S.E. General four-component scattering power decomposition with unitary transformation of coherency matrix. IEEE Trans. Geosci. Remote Sens. 2013, 51, 3014-3022. [CrossRef]

22. Chen, S.W.; Wang, X.S.; Xiao, S.P.; Sato, M. General Polarimetric model-based decomposition for coherency matrix. IEEE Trans. Geosci. Remote Sens. 2014, 52, 1843-1855. [CrossRef]

23. Cui, Y.; Yamaguchi, Y.; Yang, J.; Kobayashi, H.; Park, S.E.; Singh, G. On complete model-based decomposition of polarimetric SAR coherency matrix data. IEEE Trans. Geosci. Remote Sens. 2014, 52, 1991-2001. [CrossRef]

24. Lee, J.S.; Ainsworth, T.L.; Wang, Y. Generalized Polarimetric model-based decompositions using incoherent scattering models. IEEE Trans. Geosci. Remote Sens. 2014, 52, 2474-2491. [CrossRef]

25. Bhattacharya, A.; Singh, G.; Manickam, S.; Yamaguchi, Y. An adaptive general four-component scattering power decomposition with unitary transformation of coherency matrix (AG4U). IEEE Geosci. Remote Sens. Lett. 2015, 12, 2110-2114. [CrossRef]

26. Zou, B.; Zhang, Y.; Cao, N.; Minh, N.P. A four-component decomposition model for PolSAR data using asymmetric scattering component. IEEE J. Sel. Top. Appl. Earth Obs. Remote Sens. 2015, 8, 1051-1061. [CrossRef]

27. Cloude, S.R.; Pottier, E. An entropy based classification scheme for land applications of polarimetric SAR. IEEE Trans. Geosci. Remote Sens. 1997, 35, 68-78. [CrossRef]

28. Lee, J.S.; Grunes, M.R.; Ainsworth, T.L.; Du, L.J.; Schuler, D.L.; Cloude, S.R. Unsupervised classification using polarimetric decomposition and the complex Wishart classifier. IEEE Trans. Geosci. Remote Sens. 1999, 37, 2249-2258.

29. Ferro-Famil, L.; Pottier, E.; Lee, J. Unsupervised classification of multifrequency and fully polarimetric SAR images based on the H/A/Alpha-Wishart classifier. IEEE Trans. Geosci. Remote Sens. 2001, 39, 2332-2342. [CrossRef]

30. Shimoni, M.; Borghys, D.; Heremans, R.; Perneel, C.; Acheroy, M. Fusion of PolSAR and PolInSAR data for land cover classification. Int. J. Appl. Earth Obs. Geoinf. 2009, 11, 169-180. [CrossRef]

31. Qi, Z.; Yeh, A.G.; Li, X.; Lin, Z. A novel algorithm for land use and land cover classification using RADARSAT-2 polarimetric SAR data. Remote Sens. Environ. 2012, 118, 21-39. [CrossRef]

32. Antropov, O.; Rauste, Y.; Astola, H.; Praks, J.; Hame, T.; Hallikainen, M.T. Land cover and soil type mapping from spaceborne PolSAR data at L-band with probabilistic neural network. IEEE Trans. Geosci. Remote Sens. 2014, 52, 5256-5270. [CrossRef]

33. Wang, W.; Ji, Y.; Lin, X. A novel fusion-based ship detection method from Pol-SAR images. Sensors 2015, 15, 25072-25089. [CrossRef] [PubMed]

34. Zhang, X.; Dierking, W.; Zhang, J.; Meng, J. A polarimetric decomposition method for ice in the Bohai Sea using C-band PolSAR data. IEEE J. Sel. Top. Appl. Earth Obs. Remote Sens. 2015, 8, 47-66. [CrossRef]

35. Xiang, D.; Tang, T.; Hu, C.; Fan, Q.; Su, Y. Built-up area extraction from PolSAR imagery with model-based decomposition and polarimetric coherence. Remote Sens. 2016, 8, 685. [CrossRef]

36. Xiang, D.; Tang, T.; Ban, Y.; Su, Y.; Kuang, G. Unsupervised polarimetric SAR urban area classification based on model-based decomposition with cross scattering. ISPRS J. Photogramm. Remote Sens. 2016, 116, 86-100. [CrossRef]

37. Jagdhuber, T.; Hajnsek, I.; Papathanassiou, K.P. An iterative generalized hybrid decomposition for soil moisture retrieval under vegetation cover using fully polarimetric SAR. IEEE J. Sel. Top. Appl. Earth Obs. Remote Sens. 2015, 8, 3911-3922. [CrossRef]

38. Huang, X.D.; Wang, J.F.; Shang, J.L. An integrated surface parameter inversion scheme over agricultural fields at early growing stages by means of C-band polarimetric RADARSAT-2 imagery. IEEE Trans. Geosci. Remote Sens. 2016, 54, 2510-2528. [CrossRef]

39. Di Martino, G.; Iodice, A.; Natale, A.; Riccio, D. Polarimetric two-scale two-component model for the retrieval of soil moisture under moderate vegetation via L-band SAR data. IEEE Trans. Geosci. Remote Sens. 2016, 54, 2470-2491. [CrossRef] 
40. He, L.; Panciera, R.; Tanase, M.A.; Walker, J.P.; Qin, Q. Soil moisture retrieval in agricultural fields using adaptive model-based polarimetric decomposition of SAR data. IEEE Trans. Geosci. Remote Sens. 2016, 54, 4445-4460. [CrossRef]

41. Hajnsek, I.; Pottier, E.; Cloude, S.R. Inversion of surface parameters from polarimetric SAR. IEEE Trans. Geosci. Remote Sens. 2003, 41, 727-744. [CrossRef]

42. Hajnsek, I.; Jagdhuber, T.; Schon, H.; Papathanassiou, K.P. Potential of estimating soil moisture under vegetation cover by means of PolSAR. IEEE Trans. Geosci. Remote Sens. 2009, 47, 442-454. [CrossRef]

43. Coleman, T.F.; Li, Y. On the convergence of reflective newton methods for large-scale nonlinear minimization subject to bounds. Math. Program. 1994, 67, 189-224. [CrossRef]

44. Coleman, T.F.; Li, Y. An interior, trust region approach for nonlinear minimization subject to bounds. SIAM J. Optim. 1996, 6, 418-445. [CrossRef]

45. Ulaby, F.T.; Jedlicka, R.P. Microwave dielectric properties of plant materials. IEEE Trans. Geosci. Remote Sens. 1984, 4, 406-415. [CrossRef]

46. Orfanidis, S.J. Electromagnetic Waves and Antennas; Rutgers University: Piscataway, NJ, USA, 2016.

47. Sisser, F.S. Elimination of bounds in optimization problems by transforming variables. Math. Program. 1981, 20, 110-121. [CrossRef]

48. Gill, P.E.; Murray, W.; Saunders, M.A.; Wright, M.H. Aspects of mathematical modelling related to optimization. Appl. Math. Model. 1981, 5, 71-83. [CrossRef]

49. European Space Agency. AGRISAR 2006 Agricultural Bio-/Geophysical Retrievals from Frequent Repeat SAR and Optical Imaging Final Report; DLR: Oberpfaffenhofen, Germany, 2008. Available online: https:/ / earth.esa.int/ c/document_library/get_file?folderId=21020\&name=DLFE-397.pdf (accessed on 1 December 2015).

(C) 2016 by the authors; licensee MDPI, Basel, Switzerland. This article is an open access article distributed under the terms and conditions of the Creative Commons Attribution (CC-BY) license (http://creativecommons.org/licenses/by/4.0/). 